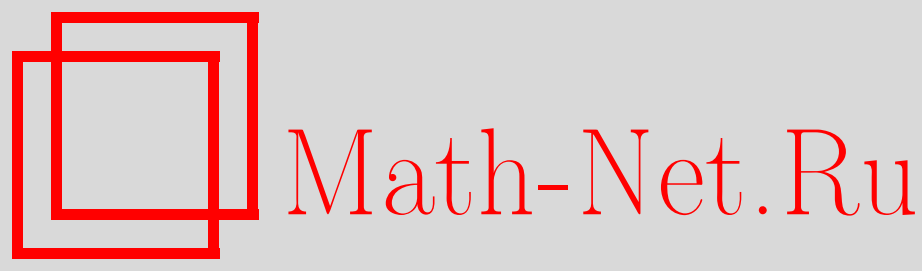

А. Ф. Барабанов, А. В. Михеенков, А. В. Шварцберг, Фрустрированный $J_{1^{-}} J_{2^{-}} J_{3}$ квантовый двумерный антиферромагнетик в сферически-симметричном самосогласованном подходе, ТМФ, 2011, том 168, номер 3, 389-416

DOI: https://doi.org/10.4213/tmf6689

Использование Общероссийского математического портала Math-Net.Ru подразумевает, что вы прочитали и согласны с пользовательским соглашением http://www . mathnet.ru/rus/agreement

Параметры загрузки:

IP : 54.92 .164 .108

26 апреля 2023 г., 17:51:05

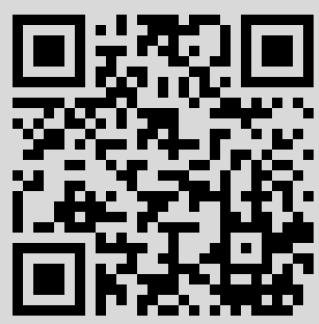




\title{
ФИЗИКА
}

Том 168, № 3

сентябрь, 2011

(C) 2011 г.

А. Ф. Барабанов*, А. В. Михеенков*†,

А. В. Шварцберг ${ }^{* \dagger}$

\section{ФРУСТРИРОВАННЫЙ $J_{1}-J_{2}-J_{3}$ КВАНТОВЫЙ ДВУМЕРНЫЙ АНТИФЕРРОМАГНЕТИК В СФЕРИЧЕСКИ-СИММЕТРИЧНОМ САМОСОГЛАСОВАННОМ ПОДХОДЕ}

\begin{abstract}
В рамках сферически-симметричного самосогласованного подхода для двухвременных запаздывающих спин-спиновых функций Грина развивается теория двумерного фрустрированного $J_{1-} J_{2}-J_{3}$ квантового $S=1 / 2$ антиферромагнетика. Показано, что учет затухания спиновых флуктуаций является определяющим при формировании как спин-жидкостного состояния, так и состояния с дальним порядком. В частности, наличие затухания позволяет объяснить скейлинговое поведение восприимчивости $\chi(\mathbf{q}, \omega)$ плоскости купратов $\mathrm{CuO}_{2}$, поведение спинового спектра в двухплоскостной ситуации и возникновение несоизмеримых $\chi(\mathbf{q}, \omega)$-пиков. В случае полной модели $J_{1}-J_{2}-J_{3}$ в рамках единого аналитического подхода найдены непрерывные переходы между тремя фазами с дальним порядком (“шахматная", страйп-фаза и геликоид $(q, q))$ через спин-жидкостное состояние. Для модели $J_{1}-J_{2}-J_{3}$ получено хорошее согласие с кластерными расчетами, для модели купратов $J_{1}-J_{2}-$ согласие с данными по нейтронному рассеянию.
\end{abstract}

Ключевые слова: высокотемпературная сверхпроводимость, низкоразмерный антиферромагнетизм, спиновая жидкость, квантовый фазовый переход.

\section{1. ВВЕДЕНИЕ}

Изучение двумерного фрустрированного $J_{1}-J_{2}-J_{3}$ квантового $S=1 / 2$ антиферромагнетика $(\mathrm{A} \Phi \mathrm{M})$ представляет большой теоретический и экспериментальный интерес. В рамках этой модели часто рассматривается вопрос о квантовом фазовом переходе. Квантовые флуктуации могут переводить систему в квантовое состояние спин-жидкостного типа даже при $T=0$. В спин-жидкостном состоянии $S U(2)$-симметрия гамильтониана восстанавливается. Вблизи точки квантового фазового перехода $\left(J_{2}=0.5 J_{1}, J_{3}=0\right)$ остается открытым вопрос о наличии фаз с нарушением трансляционной симметрии.

* Институт физики высоких давлений РАН, Троицк, Московская обл., Россия. E-mail: abarabanov@mtu-net.ru,mikheen@bk.ru

${ }^{\dagger}$ Московский физико-технический институт, Долгопрудный, Московская обл., Россия 
С экспериментальной точки зрения модель описывает слоистые квазидвумерные соединения - купраты (см., например, [1]) и ванадаты [2]-[4], при этом могут реализовываться как чисто АФМ-случаи $\left(J_{1}, J_{2}, J_{3}>0\right)$, так и случаи ферромагнитных констант связи.

Эксперименты с высокотемпературной сверхпроводимостью в купратах, в первую очередь фотоэлектронная спектроскопия с угловым разрешением и нейтронные измерения, демонстрируют множество аномальных свойств двумерного допированного АФМ, как спиновой восприимчивости $\chi(\mathbf{q}, \omega)$, так и электронной подсистемы с изменением величины допирования $x$ и $T$ [5], [6].

При рассмотрении двумерного АФМ, в том числе и допированного, активно используются различные варианты сферически-симметричной теории Кондо-Ямадзи-Шимахары-Такады [7]-[9], которая имеет ряд преимуществ относительно других подходов при описании $\chi(\mathbf{q}, \omega)$ : позволяет учесть отсутствие двух подрешеток, возникновение спиновой щели $\Delta$ при любой малой температуре $T \neq 0$ и описать дальний порядок (LRO) при $T=0$. Известно, что с увеличением допирования в двумерных АФМ спиновая корреляционная длина падает. K аналогичному поведению приводит введение фрустрации. Очевидно, что прямое соответствие между допированием и фрустрацией отсутствует. Тем не менее качественно увеличение фрустрации можно трактовать как рост $x$. Считается, что плоскость купратов $\mathrm{CuO}_{2}$ отвечает ненулевой фрустрации даже при $x=0$ [10], и чисто спиновая модель неоднократно использовалась для анализа спинового отклика допированной плоскости $\mathrm{CuO}_{2}$ (см., например, [11]).

В настоящей работе модель $J_{1}-J_{2}-J_{3}$ изучается в рамках сферически-симметричного самосогласованного подхода (СССП) [7], [8], [12], [13] в различных его вариантах: модель $J_{1}\left(J_{2}=J_{3}=0\right)$, модель $J_{1}-J_{2}\left(J_{3}=0\right)$ - этот случай наиболее исследован в кластерных расчетах, и, наконец, модель $J_{1}-J_{2}-J_{3}-$ полная модель, которая демонстрирует целый каскад фаз. Рассмотрение основано на двухвременных температурных спиновых функциях Грина $G_{\mathbf{q}}(\omega)$ [14].

В отличие от спин-волновых двухподрешеточных подходов, в СССП основные состояния как с LRO, так и без дальнего порядка (SRO) являются синглетными, теоремы Маршалла и Мермина-Вагнера выполняются автоматически, сохраняются спиновая $S U(2)$ и трансляционная симметрии (мы не рассматриваем так называемые box (блочные) и columnar (колончатые) состояния). В частности, это означает, что среднее оператора спина на узле равно нулю, $\left\langle S_{\mathbf{n}}^{\alpha}\right\rangle=0, \alpha=x, y, z$, и что спиновые корреляторы $C_{\mathbf{r}}=\left\langle S_{\mathbf{n}+\mathbf{r}}^{\alpha} S_{\mathbf{n}}^{\alpha}\right\rangle$ не зависят от положения узла $\mathbf{n}$.

Метод позволяет определять микроскопические характеристики, которые не извлекаются из кластерных расчетов, такие как спектр спиновых возбуждений $\omega_{\mathbf{q}}$, зависимость $\Delta(T)$ и ее положение и явный вид функции $\chi(\mathbf{q}, \omega, T)$.

Главное отличие нашего метода от стандартной реализации СССП заключается в том, что мы выходим за рамки среднеполевого приближения путем введения затухания в выражение для спиновой функции Грина $G_{\mathbf{q}}(\omega)$ [15].

Очевидно, вблизи линий фазовых переходов затухание спиновых флуктуаций существенно, именно его учет приводит к разумному совпадению полученной нами фазовой диаграммы модели $J_{1}-J_{2}-J_{3}$ с результатами кластерных расчетов. Более того, в работе показано, что только введение затухания в функцию $G_{\mathbf{q}}(\omega)$ позволяет описать ряд важных свойств спиновой подсистемы купратов. 
В разделе 2 , п. 2.1 вводится гамильтониан модели $J_{1}-J_{2}-J_{3}$ и для случая $J_{3}=0$ подробно представляется схема СССП обычно используемого приближения среднего поля (расцепление). Далее в п. 2.2 обсуждается возможность выхода за рамки этого приближения, получен ряд видов функции $\chi(\mathbf{q}, \omega)=-G_{\mathbf{q}}(\omega)$ и вводится основное используемое приближение для функций Грина, которое учитывает мнимую часть поляризационного оператора $M(\mathbf{q}, \omega)=M^{\prime}(\mathbf{q}, \omega)+i M^{\prime \prime}(\mathbf{q}, \omega)$ в виде $M^{\prime \prime}(\mathbf{q}, \omega)=-\omega \gamma$, где $\gamma$ - параметр затухания.

В разделе 3, п. 3.1 обсуждаются реалистические значения параметра $\gamma$ в зависимости от параметра фрустрации $p$ и температуры $T$, представлены характерные спектры, спиновые корреляторы, влияние $p$ и $T$ на величину спиновой щели, и, наконец, показывается определяющая роль спинового констрейнта при анализе влияния $\gamma$ на состояние системы. Необходимость учета затухания демонстрируется для плоскости купратов $\mathrm{CuO}_{2}$ на примерах скейлингового поведения $\chi(\mathbf{q}, \omega, T)$ (п. 3.2), оптической и акустической ветвей $\omega_{\mathbf{q}}$ двухплоскостной модели и на примере несоизмеримых пиков $\chi(\mathbf{q}, \omega)$ (п. 3.3). В разделе 4 рассматривается фазовая диаграмма модели $J_{1}-J_{2}-J_{3}$ со стороны спин-жидкостной фазы и проводится сравнение с известными кластерными данными. В разделе 5 кратко формулируются результаты и обсуждаются возможности развития СССП.

\section{2. СФЕРИЧЕСКИ-СИММЕТРИЧНЫЙ САМОСОГЛАСОВАННЫЙ ПОДХОД ДЛЯ МОДЕЛИ $J_{1}-J_{2}-J_{3}$}

2.1. Гамильтониан и приближение среднего поля. Гамильтониан фрустрированной $S=1 / 2$ модели $J_{1}-J_{2}-J_{3}$ Гейзенберга на двумерной квадратной решетке в стандартных обозначениях имеет вид

$$
\widehat{H}=\frac{1}{2} J_{1} \sum_{\mathbf{i}, \mathbf{g}} \overrightarrow{\mathbf{S}}_{\mathbf{i}} \overrightarrow{\mathbf{S}}_{\mathbf{i}+\mathbf{g}}+\frac{1}{2} J_{2} \sum_{\mathbf{i}, \mathbf{d}} \overrightarrow{\mathbf{S}}_{\mathbf{i}} \overrightarrow{\mathbf{S}}_{\mathbf{i}+\mathbf{d}}+\frac{1}{2} J_{3} \sum_{\mathbf{i}, \mathbf{g}} \overrightarrow{\mathbf{S}}_{\mathbf{i}} \overrightarrow{\mathbf{S}}_{\mathbf{i}+2 \mathbf{g}},
$$

где $\mathbf{g}, \mathbf{d}$ и $2 \mathbf{g}$ - векторы первых, вторых и третьих ближайших соседей соответственно, все обменные константы антиферромагнитные.

Ниже при рассмотрении случая $J_{3}=0$ используется стандартная переменная $p-$ параметр фрустрации $p=J_{2} / J, J_{1}=(1-p) J, J_{2}=p J, J=J_{1}+J_{2}$, и в этом случае все энергетические величины измеряются в единицах $J$, далее $J$ положено равным единице.

Вычисления проводятся в рамках СССП [8], [12], [13]. Запишем уравнение движения для функции Грина

$$
G_{\mathbf{n m}}=\left\langle S_{\mathbf{n}}^{z} \mid S_{\mathbf{m}}^{z}\right\rangle_{\omega+i \delta}=-i \int_{0}^{\infty} d t e^{i \omega t}\left\langle\left[S_{\mathbf{n}}^{z}(t), S_{\mathbf{m}}^{z}\right]\right\rangle
$$

Из-за сферической симметрии не равны нулю только диагональные по индексу $\alpha=x, y, z$ функции Грина, и имеются три вырожденные по $\alpha$ ветви спиновых возбуждений. Учитывая, что $\left\langle\left[S_{\mathbf{n}}^{z}, S_{\mathbf{m}}^{z}\right]\right\rangle=0$ и $\left[S_{\mathbf{n}}^{\alpha}, S_{\mathbf{m}}^{\beta}\right]=i \delta_{\mathbf{n m}} \varepsilon_{\alpha \beta \gamma} S_{\mathbf{n}}^{\gamma}$, для $G_{\mathbf{n m}}$ имеем

$\omega G_{\mathbf{n m}}=\left\langle\left[S_{\mathbf{n}}^{z}, S_{\mathbf{m}}^{z}\right]\right\rangle+\left\langle\left[S_{\mathbf{n}}^{z}, \widehat{H}\right] \mid S_{\mathbf{m}}^{z}\right\rangle_{\omega+i \delta}=i \sum_{\mathbf{b}=\mathbf{g}, \mathbf{d}, 2 \mathbf{g}} J_{\mathbf{b}}\left\langle\left(S_{\mathbf{n}+\mathbf{b}}^{x} S_{\mathbf{n}}^{y}-S_{\mathbf{n}+\mathbf{b}}^{y} S_{\mathbf{n}}^{x}\right) \mid S_{\mathbf{m}}^{z}\right\rangle_{\omega+i \delta}$, 
где $J_{g}=J_{1}, J_{d}=J_{2}, J_{2 g}=J_{3}$. Второй шаг дифференцирования приводит к трехузельным функциям Грина:

$$
\begin{aligned}
i \omega & \sum_{\mathbf{b}=\mathbf{g}, \mathbf{d}, 2 \mathbf{g}} J_{\mathbf{b}}\left\langle\left(S_{\mathbf{n}+\mathbf{b}}^{x} S_{\mathbf{n}}^{y}-S_{\mathbf{n}+\mathbf{b}}^{y} S_{\mathbf{n}}^{x}\right) \mid S_{\mathbf{m}}^{z}\right\rangle_{\omega+i \delta}= \\
= & 2 \sum_{\mathbf{b}=\mathbf{g}, \mathbf{d}, 2 \mathbf{g}} J_{\mathbf{b}} C_{\mathbf{b}}\left(\delta_{\mathbf{n}+\mathbf{b}, \mathbf{m}}-\delta_{\mathbf{n}, \mathbf{m}}\right)-\frac{1}{2} \sum_{\mathbf{b}=\mathbf{g}, \mathbf{d}, 2 \mathbf{g}} J_{\mathbf{b}}^{2}\left(G_{\mathbf{n}+\mathbf{b}, \mathbf{m}}-G_{\mathbf{n} \mathbf{m}}\right)- \\
& -\sum_{\substack{\mathbf{b}, \mathbf{b}^{\prime}=\mathbf{g}, \mathbf{d}, 2 \mathbf{g} ; \\
\alpha \neq z ; \mathbf{b}+\mathbf{b}^{\prime} \neq 0}}\left\{J _ { \mathbf { b } } J _ { \mathbf { b } ^ { \prime } } \left[\left\langle S_{\mathbf{n}+\mathbf{b}}^{\alpha} S_{\mathbf{n}-\mathbf{b}^{\prime}}^{z} S_{\mathbf{n}}^{\alpha} \mid S_{\mathbf{m}}^{z}\right\rangle_{\omega+i \delta}-\left\langle S_{\mathbf{n}+\mathbf{b}}^{\alpha} S_{\mathbf{n}-\mathbf{b}^{\prime}}^{\alpha} S_{\mathbf{n}}^{z} \mid S_{\mathbf{m}}^{z}\right\rangle_{\omega+i \delta}+\right.\right. \\
& \left.\left.+\left\langle S_{\mathbf{n}+\mathbf{b}}^{z} S_{\mathbf{n}+\mathbf{b}+\mathbf{b}^{\prime}}^{\alpha} S_{\mathbf{n}}^{\alpha} \mid S_{\mathbf{m}}^{z}\right\rangle_{\omega+i \delta}-\left\langle S_{\mathbf{n}+\mathbf{b}}^{\alpha} S_{\mathbf{n}+\mathbf{b}+\mathbf{b}^{\prime}}^{z} S_{\mathbf{n}}^{\alpha} \mid S_{\mathbf{m}}^{z}\right\rangle_{\omega+i \delta}\right]\right\},
\end{aligned}
$$

где $C_{\mathbf{b}}=\left\langle S_{\mathbf{n}+\mathbf{b}}^{z} S_{\mathbf{n}}^{z}\right\rangle$.

В среднеполевом подходе дальнейшая процедура сводится к замыканию цепочки уравнений движения на втором шаге с помощью расцепления трехузельных членов, которое имеет следующий характерный вид:

$$
\begin{aligned}
S_{\mathbf{n}+\mathbf{g}_{1}+\mathbf{g}_{2}}^{j} S_{\mathbf{n}+\mathbf{g}_{1}}^{l} S_{\mathbf{n}}^{\gamma} \approx & \alpha_{\mathbf{g}}\left(\delta_{j l}\left\langle S_{\mathbf{n}+\mathbf{g}_{1}+\mathbf{g}_{2}}^{j} S_{\mathbf{n}+\mathbf{g}_{1}}^{l}\right\rangle S_{\mathbf{n}}^{\gamma}+\delta_{l \gamma}\left\langle S_{\mathbf{n}+\mathbf{g}_{1}}^{l} S_{\mathbf{n}}^{\gamma}\right\rangle S_{\mathbf{n}+\mathbf{g}_{1}+\mathbf{g}_{2}}^{j}\right)+ \\
& +\alpha_{\mathbf{g}_{1}+\mathbf{g}_{2}} \delta_{j \gamma}\left\langle S_{\mathbf{n}+\mathbf{g}_{1}+\mathbf{g}_{2}}^{j} S_{\mathbf{n}}^{\gamma}\right\rangle S_{\mathbf{n}+\mathbf{g}_{1}}^{l},
\end{aligned}
$$

где $\alpha_{\mathbf{g}}, \alpha_{\mathbf{g}_{1}+\mathbf{g}_{2}}-$ вершинные поправки. Подчеркнем, что в нашем случае $(S=1 / 2)$ процедура расцепления для каждого члена однозначна, поскольку $\alpha \neq z$, т. е. приводит к одному члену (в среднее выносятся два спина с $\alpha \neq z$ ).

После перехода к фурье-образу

$$
S_{\mathbf{q}}^{z}=\frac{1}{\sqrt{N}} \sum_{\mathbf{r}} e^{-i \mathbf{q r}} S_{\mathbf{r}}^{z}
$$

решение для двух уравнений приводит к ответу для среднеполевой функции Грина $G_{\mathrm{mf}}(\mathbf{q}, \omega)=\left\langle S_{\mathbf{q}}^{z} \mid S_{-\mathbf{q}}^{z}\right\rangle_{\omega}=-\chi_{\mathrm{mf}}(\mathbf{q}, \omega)$,

$$
G_{\mathrm{mf}}(\mathbf{q}, \omega)=\frac{F_{\mathbf{q}}}{\omega^{2}-\omega_{\mathbf{q}}^{2}} .
$$

Выпишем $F_{\mathbf{q}}$ и $\omega_{\mathbf{q}}^{2}$ для случая $J_{3}=0$ (полные выражения при $J_{3} \neq 0$ несложно вычисляются, но имеют громоздкий вид, и мы их не приводим):

$$
\begin{gathered}
F_{\mathbf{q}}=-8\left[J_{1} C_{g}\left(1-\beta_{g}\right)+J_{2} C_{d}\left(1-\beta_{d}\right)\right], \\
\omega_{\mathbf{q}}^{2}=2\left[\left(\beta_{1} K_{1}+\beta_{2} K_{2}\right)-\left(\beta_{3} K_{3}+\beta_{4} K_{4}\right)-\left(\beta_{5} K_{5}+\beta_{6} K_{6}\right)\right] .
\end{gathered}
$$

Входящие в выражение для спектра величины $K_{1}, \ldots, K_{6}$ равны

$$
\begin{gathered}
K_{1}=J_{1} J_{2} K_{g d}+12 J_{1}^{2} \widetilde{C}_{g}+1+K_{g g}, \quad K_{2}=J_{1} J_{2} K_{g d}+12 J_{2}^{2} \widetilde{C}_{d}+1+K_{d d}, \\
K_{3}=16 J_{1}^{2} \widetilde{C}_{g}, \quad K_{4}=16 J_{2}^{2} \widetilde{C}_{d}, \quad K_{5}=16 J_{1} J_{2} \widetilde{C}_{g}, \quad K_{6}=16 J_{1} J_{2} \widetilde{C}_{d}, \\
K_{g g}=\sum_{\substack{\mathbf{r}=\mathbf{g}_{1}+\mathbf{g}_{2} ; \\
\mathbf{g}_{1} \neq-\mathbf{g}_{2}}} \widetilde{C}_{r}, \quad K_{d d}=\sum_{\substack{\mathbf{r}=\mathbf{d}_{1}+\mathbf{d}_{2} ; \\
\mathbf{d}_{1} \neq-\mathbf{d}_{2}}} \widetilde{C}_{r}, \quad K_{g d}=\sum_{\mathbf{r}=\mathbf{g}+\mathbf{d}} \widetilde{C}_{r}, \\
\beta_{1}=1-\beta_{g}, \quad \beta_{2}=1-\beta_{d}, \quad \beta_{3}=1-\beta_{g}^{2}, \quad \beta_{4}=1-\beta_{d}^{2}, \quad \beta_{5}=\left(1-\beta_{g}\right) \beta_{d}, \\
\beta_{6}=\left(1-\beta_{d}\right) \beta_{g}, \quad \beta_{g}=\frac{1}{2}\left(\cos q_{x}+\cos q_{y}\right), \quad \beta_{d}=\cos q_{x} \cos q_{y},
\end{gathered}
$$


здесь $\widetilde{C}_{r}$ отвечают корреляторам $C_{r}$, которые перенормированы упомянутыми вершинными поправками $\alpha_{\mathbf{r}}$.

В функцию Грина $G(\mathbf{q}, \omega)$ входят корреляторы $C_{\mathbf{r}}$ для первых пяти координационных сфер $\mathbf{r}=\mathbf{g}, \mathbf{d}, 2 \mathbf{g}, 2 \mathbf{d}, \mathbf{g}+\mathbf{d}$, которые должны вычисляться самосогласованно через $G(\mathbf{q}, \omega)$. Кроме того, для $G(\mathbf{q}, \omega)$ должен выполняться спиновый констрейнт $C_{\mathbf{r}=0}=\left\langle S_{\mathbf{i}}^{z} S_{\mathbf{i}}^{z}\right\rangle=1 / 4$ (правило сумм). Эти условия имеют вид

$$
\begin{aligned}
C_{\mathbf{r}} & =\frac{1}{N} \sum_{\mathbf{q}} C_{\mathbf{q}} e^{i \mathbf{q r}}, \quad C_{\mathbf{q}}=\left\langle S_{\mathbf{q}}^{z} S_{-\mathbf{q}}^{z}\right\rangle=-\frac{1}{\pi} \int_{0}^{\infty} d \omega(2 m(\omega)+1) G^{\prime \prime}(\mathbf{q}, \omega), \\
C_{\mathbf{r}=0} & =\frac{1}{4}=-\frac{1}{\pi} \frac{1}{N} \sum_{\mathbf{q}} \int_{0}^{\infty} d \omega(2 m(\omega)+1) G^{\prime \prime}(\mathbf{q}, \omega), \quad G^{\prime \prime}=\operatorname{Im} G(\mathbf{q}, \omega) .
\end{aligned}
$$

Система самосогласованных уравнений затем решается численно.

В среднеполевом случае мнимая часть спиновой динамической восприимчивости имеет вид

$$
\chi_{\mathrm{mf}}^{\prime \prime}(\mathbf{q}, \omega)=\frac{\pi F_{\mathbf{q}}}{2 \omega_{\mathbf{q}}}\left[\delta\left(\omega-\omega_{\mathbf{q}}\right)-\delta\left(\omega+\omega_{\mathbf{q}}\right)\right] .
$$

В общем случае в рамках модели (2), (3) могут реализовываться как SRO-состояния, так и LRO-состояния. Благодаря двумерности LRO-состояния могут существовать только в пределе $T \rightarrow 0$ и характеризуются тем, что спин-спиновые корреляторы на бесконечности $\left\langle S_{\mathbf{r}}^{\alpha} S_{\mathbf{0}}^{\alpha}\right\rangle_{r \rightarrow \infty}$ отличны от нуля.

При $T \neq 0$ в спектре спиновых возбуждений всегда есть щель. При малых $T$ функция $C_{\mathbf{q}}$ всегда имеет пик около точек $\mathbf{q}_{0}$, где минимальна спиновая щель (кроме тривиальной нулевой точки, для которой $\left.F_{\mathbf{q}=0}=0\right)$.

При $T \rightarrow 0$ возможны две ситуации. В первом случае система остается в SRO-cocтоянии (в состоянии спиновой жидкости), щель не закрывается и вклад от $m(\omega)$ в $C_{\mathbf{q}}(9)$ становится равным нулю при $T \rightarrow 0$ (случай фрустрации, близкой к $p=0.3$ для модели $\left.J_{1}-J_{2}\right)$.

Во втором случае при понижении температуры система переходит в LRO-cocтояние и щель закрывается в точке $\mathbf{q}_{0}$, в которой для модели $J_{1}-J_{2}$ может быть либо фаза $\mathbf{q}_{0}^{\mathrm{N}}=\mathbf{Q}=(\pi, \pi)$ - дальний порядок неелевского типа (NLRO), малая фрустрация, либо фаза $\mathbf{q}_{0}^{\mathrm{S}}=\mathbf{X}=(\pi, 0) ;(0, \pi)$ - дальний порядок страйп-типа (StLRO), большая фрустрация. NLRO-состоянию с дальним порядком отвечают АФМ-корреляторы с "шахматным" мотивом $\left\langle S_{\mathbf{r}}^{\alpha} S_{\mathbf{0}}^{\alpha}\right\rangle_{r \rightarrow \infty}=c_{0}(-1)^{n_{x}+n_{y}}\left(\mathbf{r}=n_{x} \mathbf{g}_{x}+\right.$ $n_{y} \mathbf{g}_{y}$, где $\mathbf{g}_{x}=(1,0), \mathbf{g}_{y}=(0,1)$ - векторы ближайших соседей) [8], и такое состояние является синглетным аналогом двухподрешеточного неелевского ("шахматного") состояния.

При этом при $T \rightarrow 0$ часть размытой спектральной интенсивности структурного фактора $C_{\mathbf{q}}$ переходит в $\delta$-образную интенсивность, которая определяет конденсат. Вклада от члена без бозевской функции не хватает, чтобы обеспечить условие констрейнта. В этом случае спектральная плотность имеет вид

$$
I_{\mathbf{q}}(\omega)=-\frac{1}{\pi} \theta(\omega) G_{\mathbf{q}}^{\prime \prime}(\omega)+c_{0} \frac{1}{\nu} \sum_{i=1}^{\nu} \delta(\omega) \Delta_{\mathbf{q}, \mathbf{q}_{0}^{\nu}},
$$

где $c_{0}$ - величина конденсата, а $\nu$ - число неэквивалентных точек $\mathbf{q}_{0}^{\nu}$, в которых обращается в нуль спектр спиновых флуктуаций: при малых $p$ это одна точка $\mathbf{Q}$, 


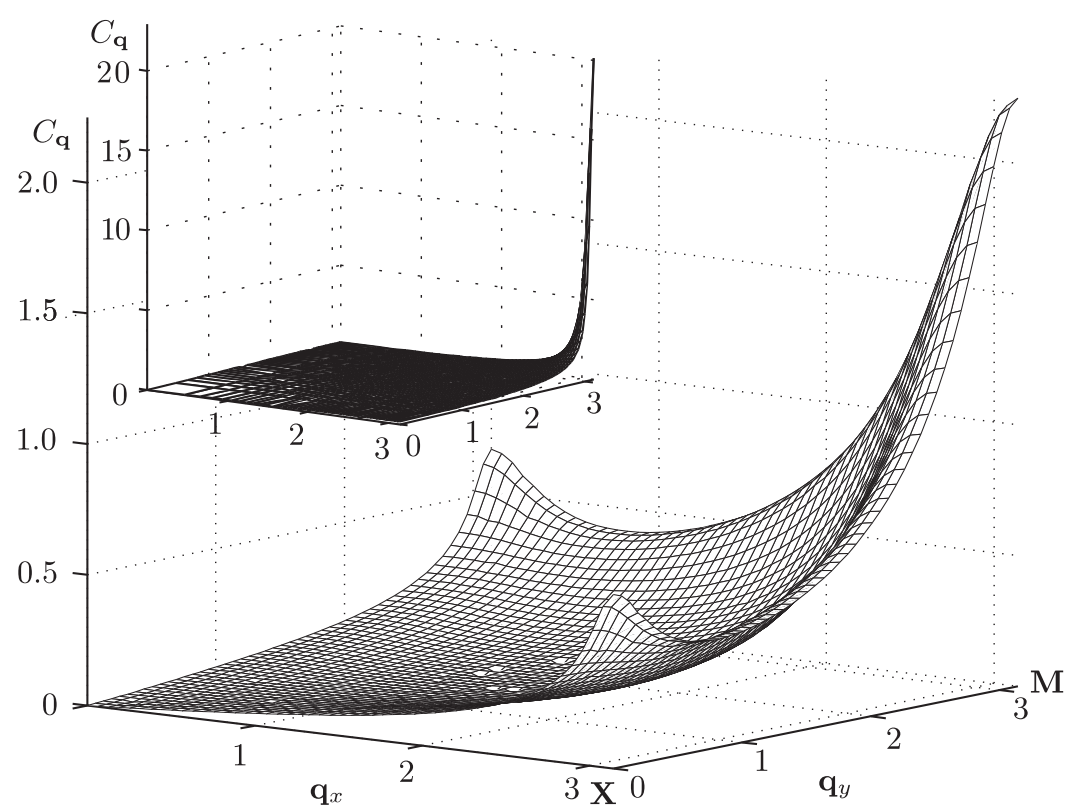

Рис. 1. Структурный фактор $C_{\mathbf{q}}$ при промежуточной фрустрации $p=0.28$ (проявляются одновременно мотивы NLRO и StLRO). Для сравнения на вставке показан узкий пик $C_{\mathbf{q}}$ при малой фрустрации $p=0.1$ (только мотив NLRO).

при $p$, близком к единице, в страйп-фазе, это две точки $(\pi, 0)$ и $(0, \pi)$. При этом в СССП страйп-фаза представляет собой не классическую структуру из чередующихся полос спинов вверх и спинов вниз, а когерентную квантовую суперпозицию горизонтальных и вертикальных полос [12], [13]. В обоих пределах существует LRO, и спиновые корреляторы на больших расстояниях имеют вид

$$
\begin{gathered}
\left\langle S_{\mathbf{r}}^{\alpha} S_{\mathbf{0}}^{\alpha}\right\rangle_{r \rightarrow \infty}^{\mathrm{N}}=c_{0}^{\mathrm{N}}(-1)^{n_{x}+n_{y}}, \quad\left\langle S_{\mathbf{r}}^{\alpha} S_{\mathbf{0}}^{\alpha}\right\rangle_{r \rightarrow \infty}^{\mathrm{S}}=\frac{c_{0}^{\mathrm{S}}}{2}\left[(-1)^{n_{x}}+(-1)^{n_{y}}\right], \\
\mathbf{r}=n_{x} \mathbf{g}_{x}+n_{y} \mathbf{g}_{y} .
\end{gathered}
$$

При конечной температуре щель открывается, LRO исчезает, $C_{\mathbf{r}}$ переходит в обращающуюся в нуль на бесконечности убывающую функцию, структурный фактор размывается на величину порядка обратной корреляционной длины $\xi^{-1}$. В области же промежуточных фрустраций, во-первых, при $T=0$ LRO отсутствует, во-вторых, "мотивы" неелевской и страйп-фаз присутствуют с сопоставимыми весами. Поэтому структурный фактор $C_{\mathbf{q}}$ представляет собой суперпозицию размытых пиков вокруг точек $\mathbf{Q}$ и $\mathbf{X}$ с переходными участками между ними. Характерный вид такого поведения $C_{\mathbf{q}}(p)$ представлен на рис. 1 для $p=0.28, T=0.025$ и $p=0.1, T=0.1$ (низкие температуры).

Как отмечалось, в выражениях $(7),(8) \widetilde{C}_{r}=\alpha_{r} C_{r}$ - корреляторы с учетом вершинных поправок. Вершинные поправки могут быть различными для разных координационных сфер. В работе [8] в модели без фрустрации принималась схема, 
в которой для ближайших соседей $\alpha_{r=g}=\alpha_{1}$, а на дальних (второй и третьей) координационных сферах $(r>g) \alpha_{r}=\alpha_{2}$. В модели с фрустрацией, $J_{1}, J_{2}>0, J_{3}=0$, разумно использовать следующую схему для вершинных поправок [12]: для ближайших соседей $\alpha_{r=g}=\alpha_{1}$; на дальних (третьей, четвертой и пятой) координационных сферах $\alpha_{r>d}=\alpha_{2}$; в промежуточном случае вторых соседей $(r=d)$ принимается $\alpha_{r=d}=\alpha_{3}$. Таким образом, возникают три различных вершинных поправки, введение которых обеспечивает выполнение трех дополнительных условий.

Первое условие - спиновый констрейнт $C_{\mathbf{r}=0}=\left\langle S_{\mathbf{n}}^{\alpha} S_{\mathbf{n}}^{\alpha}\right\rangle=1 / 4$. В качестве второго условия используется соотношение $\alpha_{3}=(1-p) \alpha_{2}+p \alpha_{1}$, которое обеспечивает корректный предел $p \rightarrow 1$, где решетка (после перехода в страйп-фазу) распадается на две невзаимодействующие АФМ-подрешетки. В частности, выполняются следующие соответствия между корреляторами $C_{\mathbf{r}}^{p=0}$ и корреляторами $C_{\mathbf{r}}^{p=1}: C_{d}^{p=1}=C_{g}^{p=0}$, $C_{2 g}^{p=1}=C_{d}^{p=0}, C_{g}^{p=1}=0$. И, наконец, третье условие -

$$
r_{\alpha}=\frac{\alpha_{1}-1}{\alpha_{2}-1}=\mathrm{const}
$$

где параметр $r_{\alpha}$ считается не зависящим от температуры. Функциональный вид (13) приводит к тому, что все вершинные поправки $\alpha_{r}$ при высокой температуре стремятся к единице [8]. Величина $r_{\alpha}$ обычно выбирается так, чтобы обеспечить сшивку с пределом нулевой температуры и нулевой фрустрации, который хорошо изучен несколькими альтернативными методами [16].

При выборе $r_{\alpha}=1$, очевидно, $\alpha_{1}=\alpha_{2}=\alpha_{3}$. Характерные значения $r_{\alpha}$ обычно выбираются в интервале $r_{\alpha}=0.8-1.0$, при этом характерные значения $\alpha_{1}=1.5-1.9$.

В пределе нулевой фрустрации (модель $J_{1}$ ) имеются результаты нескольких альтернативных подходов [16], в том числе расчетов методом Монте-Карло. Последние дают для спин-спинового коррелятора на ближайших соседях оценку $C_{\mathbf{g}}=$ $-0.1115 \pm 0.0015$ и для определяемой конденсатом эффективной намагниченности $m^{N}=\sqrt{3 c_{0}}=0.304 \pm 0.004$ (использована нормировка из работ [8], [16]).

При $p=0$ СССП в приближении среднего поля не вполне согласуется с этими данными. Подбор параметра $r_{\alpha}$ позволяет воспроизвести либо одну, либо другую из указанных величин, но не обе одновременно (при $r_{\alpha}=0.852$ имеем $C_{\mathbf{g}}=-0.1117$, но $m^{N}=0.253$, при $r_{\alpha}=0.826$ получаем $m^{N}=0.300$, но $C_{\mathbf{g}}=-0.1169$ [8]). Везде ниже, если не оговорено специально, принят параметр $r_{\alpha}=0.863$. Этот выбор приводит к значению энергии на узел, равному $6 C_{\mathrm{g}}=0.66$ при $T=0, p=0$.

На рис. 2 приведена, в частности, зависимость эффективной намагниченности $m=\sqrt{3 c_{0}^{\mathrm{N}, \mathrm{S}}}$ от параметра фрустрации $p$ в среднеполевом (случай $\left.\gamma=0\right)$ приближении для модели $J_{1}-J_{2}$. В этом случае, как видно из рис. 2 , границы перехода по параметру $p$ из LRO-состояний в состояние спиновой жидкости для NLRO и для состояния StLRO равны соответственно $p_{0}^{\mathrm{N}} \approx 0.1$ и $p_{0}^{\mathrm{S}} \approx 0.62$.

На рис. 3 представлена характерная эволюция спектра спиновых возбуждений $\omega_{\mathbf{q}}$ в среднеполевом приближении с ростом фрустрации от $p=0.05$ до $p=0.3$ при $T / J=0.1$. Явно видно, что при росте $p$ щель в точке $\mathbf{Q}=(\pi, \pi)$ увеличивается, а в точках $\mathbf{X}$, отвечающих StLRO-состоянию, щель уменьшается. Можно считать, что качественно среднеполевое приближение повторяет основные черты изменения спиновой подсистемы в зависимости от фрустрации $p$. Однако, как будет видно ниже, для корректного описания модели выход за среднеполевое приближение является обязательным. 


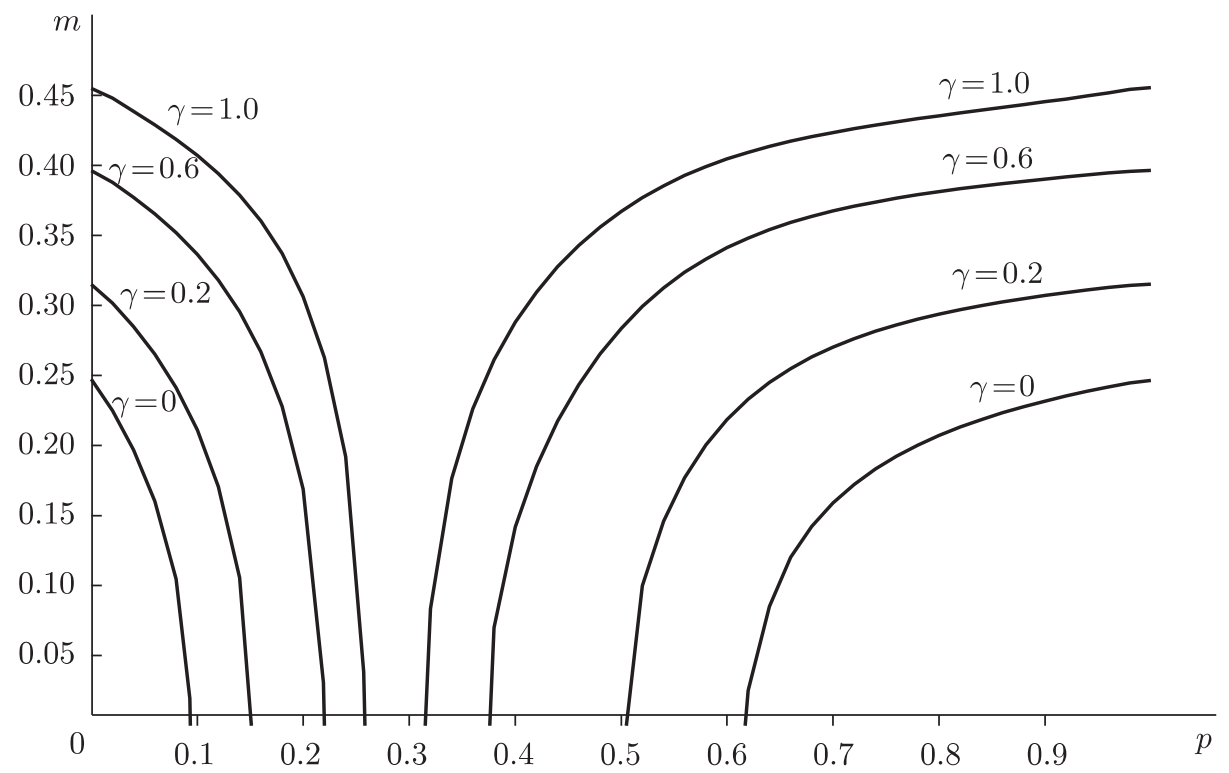

Рис. 2. Зависимость эффективной намагниченности $m=\sqrt{3 c_{0}^{\mathrm{N}, \mathrm{S}}}$ от параметра фрустрации $p$ при $T=0$ для модели $J_{1}-J_{2}$ при значениях параметра затухания $\gamma=0 ; 0.2 ; 0.6 ; 1.0\left(r_{\alpha}=0.863\right)$.

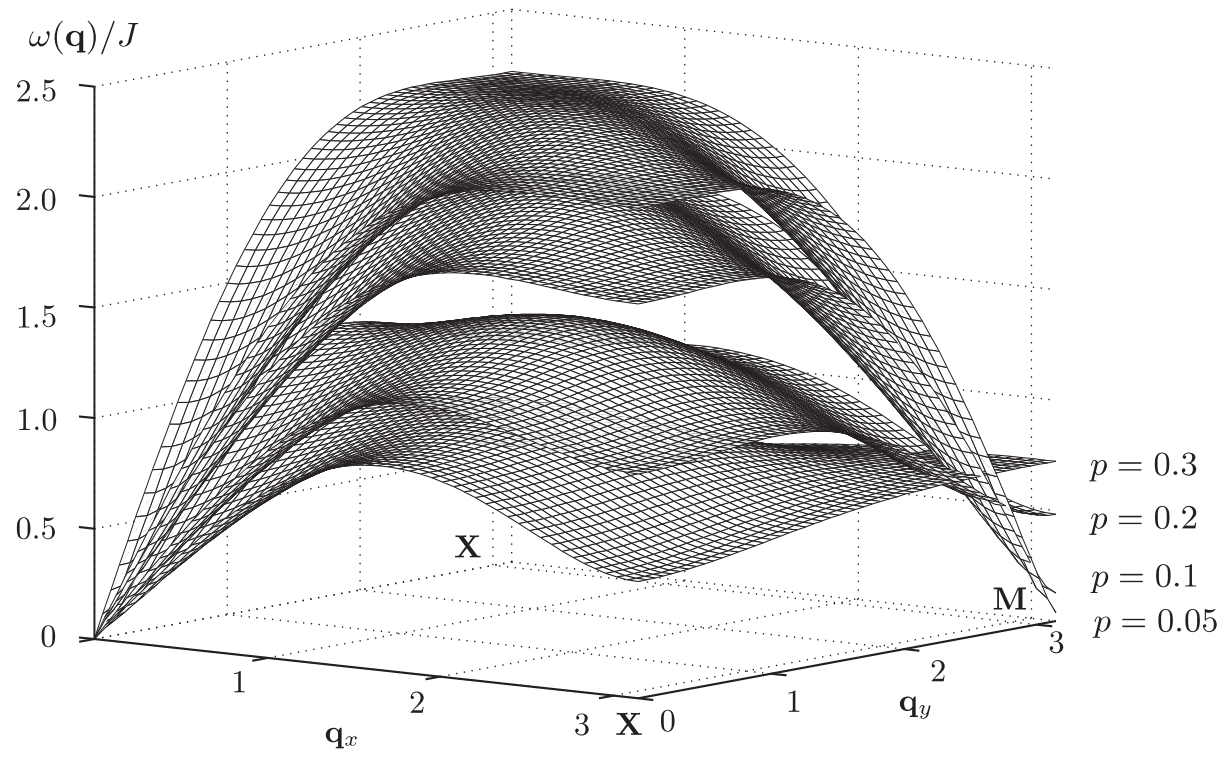

Рис. 3. Эволюция спектра спиновых возбуждений $\omega(\mathbf{q})$ в среднеполевом приближении с ростом фрустрации. Температура $T / J=0.1$. Показана четверть полной зоны Бриллюэна, $\mathbf{M} \equiv \mathbf{Q}=(\pi, \pi), \mathbf{X}=(0, \pi),(\pi, 0)$. 


\section{2. Выход за приближение среднего поля и простейший учет зату-} хания. В рамках СССП, следуя [8], [12], [17], с помощью метода неприводимых функций Грина [18], [19] (который близок к методу функций памяти [20]-[25], однако мы используем явное выражение для спектра спиновых возбуждений, что позволяет провести самосогласование) можно вывести формально точное выражение для функции Грина $\left\langle S_{\mathbf{q}}^{z} \mid S_{-\mathbf{q}}^{z}\right\rangle_{\omega}$. Оно имеет вид

$$
G(\mathbf{q}, \omega)=\frac{F_{\mathbf{q}}}{\omega^{2}-\omega_{\mathbf{q}}^{2}-M(\mathbf{q}, \omega)}=\frac{F_{\mathbf{q}}}{\omega^{2}-\widetilde{\omega}_{\mathbf{q}}^{2}+i \omega \gamma(\mathbf{q}, \omega)},
$$

где $\omega_{\mathbf{q}}$ (спектр спиновых возбуждений) и $F_{\mathbf{q}}$ имеют тот же вид, что и в приближении среднего поля $(7),(8)$, а $M(\mathbf{q}, \omega)=M^{\prime}+i M^{\prime \prime}$ - фурье-образ новой сложной функции Грина, аналитические свойства которой такие же, как у $G(\mathbf{q}, \omega)$.

Функция $M(\mathbf{q}, \omega)$ отвечает трехузельной неприводимой запаздывающей функции Грина и описывает распад спиновой волны на три составляющие (или эффективный распад спинового возбуждения на электронное и дырочное возбуждения в случае $t-J$ модели). Последнее выражение для $\chi(\mathbf{q}, \omega)$ в (14) более удобно для анализа, поскольку явно учитывает, что $M^{\prime \prime}(\mathbf{q}, \omega)=-\omega \gamma(\mathbf{q}, \omega)$ является нечетной функцией $\omega$, и легко позволяет для приближенного $\gamma(\mathbf{q}, \omega)$ добиться выполнения правила сумм. Параметр $\widetilde{\omega}_{\mathbf{q}}^{2}=\omega_{\mathbf{q}}^{2}-M_{\mathbf{q}}^{\prime}$ дает эффективный спектр спиновых волн, перенормированный функцией $M^{\prime}(\mathbf{q}, \omega)$. Здесь мы пренебрегаем зависимостью $M^{\prime}(\mathbf{q}, \omega)$ от $\omega$.

Числитель $F_{\mathbf{q}}$ и спектр спин-волновых возбуждений $\omega_{\mathbf{q}}$ выражаются через параметр фрустрации $p$ и конечное число спин-спиновых корреляционных функций, которые должны вычисляться самосогласованно.

Функции $F_{\mathbf{q}}$ и $\omega_{\mathbf{q}}$ обращаются в нуль при $\mathbf{q} \rightarrow 0$. В случае модели $J_{1}-J_{2}$ в области малых фрустраций в пределе $\mathbf{q} \rightarrow \mathbf{Q}$ числитель $F_{\mathbf{q}}$ стремится к некоторой положительной константе $F_{\mathbf{Q}}$, а спектр спиновых возбуждений $\omega_{\mathbf{q}}^{2} \approx \Delta^{2}+c^{2}(\mathbf{q}-\mathbf{Q})^{2}$ содержит щель $\Delta \equiv \omega_{\mathbf{Q}}$. Если мы учитываем $M^{\prime}(\mathbf{q}, \omega)$, мы должны использовать перенормированную щель $\tilde{\Delta}$. В области $\mathbf{q} \approx \mathbf{Q}$ спиновая восприимчивость $\chi(\mathbf{q}, \omega)$ имеет вид

$$
\chi(\mathbf{q}, \omega) \approx \frac{\chi_{\mathbf{Q}}}{1+\xi^{2}(\mathbf{q}-\mathbf{Q})^{2}-\omega^{2} \xi^{2} / c^{2}-i \omega / \omega_{\mathrm{sf}}}, \quad \chi_{\mathbf{Q}}=\frac{F_{\mathbf{Q}} \xi^{2}}{c^{2}}
$$

где мы ввели корреляционную длину $\xi=c / \tilde{\Delta}$ и параметр $\omega_{\mathrm{sf}}=\tilde{\Delta}^{2} / \gamma(\mathbf{Q}, \tilde{\Delta})$, который представляет собой характерный масштаб энергий для АФМ-флуктуаций. Аналогичная форма феноменологической спиновой восприимчивости используется в теории почти АФМ ферми-жидкости [26]-[28]. Вблизи страйп-фазы соответствующие разложения должны проводиться около точки $\mathbf{X}$.

В случае модели $J_{1}$ фурье-образ $M(\mathbf{q}, \omega)$ определяется трехузельной неприводимой функцией Грина $\left\langle\delta b_{\mathbf{q}}^{z} \mid \delta b_{-\mathbf{q}}^{z}\right\rangle_{\omega}$ с оператором $\delta b_{\mathbf{q}}^{z}$ следующего вида [17]:

$$
\begin{aligned}
\delta b_{\mathbf{q}}^{z}= & N^{-1 / 2} \sum_{\substack{\mathbf{r}, \alpha, \mathbf{g}, \mathbf{g}^{\prime} ; \\
\mathbf{g}^{\prime} \neq \mathbf{g}, \alpha \neq z}} e^{-i \mathbf{q} \mathbf{r}}\left[-\left(S_{\mathbf{r}}^{\alpha} S_{\mathbf{r}+\mathbf{g}}^{\alpha}\right)^{\mathrm{irr}} S_{\mathbf{r}+\mathbf{g}^{\prime}}^{z}+\left(S_{\mathbf{r}+\mathbf{g}}^{\alpha} S_{\mathbf{r}+\mathbf{g}^{\prime}}^{\alpha}\right)^{\mathrm{irr}} S_{\mathbf{r}}^{z}-\right. \\
& \left.-\left(S_{\mathbf{r}}^{\alpha} S_{\mathbf{r}+\mathbf{g}-\mathbf{g}^{\prime}}^{\alpha}\right)^{\mathrm{irr}} S_{\mathbf{r}+\mathbf{g}}^{z}+\left(S_{\mathbf{r}}^{\alpha} S_{\mathbf{r}+\mathbf{g}}^{\alpha}\right)^{\mathrm{irr}} S_{\left.\mathbf{r}+\mathbf{g}-\mathbf{g}^{\prime}\right]}^{z}\right]
\end{aligned}
$$


где для неприводимой части операторов приняты обозначения $\left(S_{\mathbf{r}}^{\alpha} S_{\mathbf{r}^{\prime}}^{\alpha}\right)^{\mathrm{irr}}=S_{\mathbf{r}}^{\alpha} S_{\mathbf{r}^{\prime}}^{\alpha}-$ $\left\langle S_{\mathbf{r}}^{\alpha} S_{\mathbf{r}^{\prime}}^{\alpha}\right\rangle$. Мнимая часть $M(\mathbf{q}, \omega)$ выражается как

$$
\begin{aligned}
& M^{\prime \prime}(\mathbf{q}, \omega)=\frac{2 \pi}{F_{\mathbf{q}} N} \sum_{\mathbf{k}_{1}, \mathbf{k}_{2}, \mathbf{k}_{3}} \int d \omega_{1} d \omega_{2} d \omega_{3} \delta\left(\omega-\sum_{i=1}^{3} \omega_{i}\right) \times \\
& \quad \times m\left(\omega_{1}\right) m\left(\omega_{2}\right) m\left(\omega_{3}\right)\left(1-e^{\beta \omega}\right) \Theta_{\mathbf{q}, \mathbf{k}_{1}, \mathbf{k}_{2}, \mathbf{k}_{3}}^{2} G^{\prime \prime}\left(\mathbf{k}_{1}, \omega_{1}\right) G^{\prime \prime}\left(\mathbf{k}_{2}, \omega_{2}\right) G^{\prime \prime}\left(\mathbf{k}_{3}, \omega_{3}\right), \\
& \Theta_{\mathbf{q}, \mathbf{k}_{1}, \mathbf{k}_{2}, \mathbf{k}_{3}}=16 N^{-1} \delta_{\mathbf{q}, \mathbf{k}_{1}+\mathbf{k}_{2}+\mathbf{k}_{3}}\left[\left(-\beta_{g}\left(\mathbf{k}_{2}\right) \beta_{g}\left(\mathbf{k}_{3}\right)+\beta_{g}\left(\mathbf{k}_{1}\right) \beta_{g}\left(\mathbf{k}_{2}\right)-\right.\right. \\
& \quad-\beta_{g}\left(\mathbf{k}_{2}+\mathbf{k}_{3}\right) \beta_{g}\left(\mathbf{k}_{2}\right)+\beta_{g}\left(\mathbf{k}_{2}+\mathbf{k}_{3}\right) \beta_{g}\left(\mathbf{k}_{3}\right)-4^{-1}\left(\beta_{g}\left(\mathbf{k}_{2}+\mathbf{k}_{3}\right)-\right. \\
& \left.\left.\quad-\beta_{g}\left(\mathbf{k}_{1}+\mathbf{k}_{2}\right)-\beta_{g}\left(\mathbf{k}_{3}\right)+\beta_{g}\left(\mathbf{k}_{2}\right)\right)\right] .
\end{aligned}
$$

Выражение для $M^{\prime \prime}(q, \omega)$ для фрустрированной модели должно быть более громоздким, и, насколько нам известно, явный вид его пока не получен. Сложности прямого вычисления поляризационного оператора также заключаются в том, что входящая в него функция Грина должна вычисляться самосогласованным образом. В альтернативных подходах вычисление мнимой части поляризационного оператора также затруднительно, известны только некоторые асимптотики для нефрустрированной модели при $T \rightarrow 0$ в рамках двухподрешеточного формализма Дайсона-Малеева [29]. Таким образом, последовательное вычисление $M(\mathbf{q}, \omega)$ наталкивается на существенные математические трудности [17], поэтому приходится прибегать к полуфеноменологическому рассмотрению. Ниже мы используем наше основное приближение - в выражении для функции Грина (14) учитывается только мнимая часть $M(\mathbf{q}, \omega)$ в рамках выражения $M^{\prime \prime}(\mathbf{q}, \omega)=-\omega \gamma$, т. е. $\gamma$ не зависит от $\mathbf{q}$ и $\omega$ :

$$
G(\mathbf{q}, \omega)=\frac{F_{\mathbf{q}}}{\omega^{2}-\omega_{\mathbf{q}}^{2}+i \omega \gamma}, \quad G_{\mathbf{q}}^{\prime \prime}(\mathbf{q}, \omega)=-\frac{F_{\mathbf{q}} \omega \gamma}{\left(\omega^{2}-\omega_{\mathbf{q}}^{2}\right)^{2}+\omega^{2} \gamma^{2}},
$$

при этом мы, как и в случае среднего поля, проводим полное самосогласование для всех значений $p$ и $T$. В следующем разделе мы покажем, что уже это простейшее приближение позволяет объяснить целый ряд экспериментальных результатов по сверхпроводящим купратам.

\section{3. ВЛИЯНИЕ ЗАТУХАНИЯ НА СВОЙСТВА СПИНОВОЙ СИСТЕМЫ}

\section{1. Роль затухания в формировании дальнего порядка и спиновой ще-} ли. Обсудим значения параметра затухания $\gamma$ в (15), принимаемые как в предыдущих рассмотрениях, так и в настоящей работе. Некоторые указания на порядок величины $\gamma$ во фрустрированной спиновой модели можно получить, анализируя доступные результаты для $t-J$ модели [22], [30]. При этом предполагается, что качественно увеличение допирования в модели с носителями соответствует увеличению фрустрации в чисто спиновой модели. Если рассматривать систему в спин-жидкостном состоянии, то следует сравнивать затухание с данными $t-J$ модели при таком допировании, когда LRO отсутствует. В работе [30] соответствующее допирование $\delta=0.15$ приводит к минимальному значению $\gamma \sim 0.7 J$, близкому к значениям, принимаемым нами ниже. Отметим, что к значениям $\gamma>0.7 J$ приводит экстраполяция к нулю температурной зависимости $\gamma(T)$ из данных кластер- 
ной диагонализации [22]. Детальный анализ других доступных данных приводит к диапазону оценок $\gamma$ в моделях с допированием $\gamma \sim 0.4-1.0$. Наши исследования спиновых свойств плоскости $\mathrm{CuO}_{2}$, выполненные на основе приближения (15) и приводящие к хорошему согласию с данными нейтронных экспериментов и поведением кинетических коэффициентов в высокотемпературных сверхпроводниках, приводят к аналогичному диапазону $\gamma \lesssim J[31]-[34]$. Случаю больших $\gamma \simeq 1.0 J$ может отвечать экспериментальная ситуация при сильном рассеянии спиновых флуктуаций на примесях.

Зависимость эффективной намагниченности $m=\sqrt{3 c_{0}^{\mathrm{N}, \mathrm{S}}}$ от параметра фрустрации $p$, представленная на рис. 2 , показывает, что с возрастанием $\gamma$ область спин-жидкостной фазы SRO сужается. Существенно, что относительно среднеполевого поведения с $p_{0}^{\mathrm{N}}(\gamma=0) \approx 0.1$ точка перехода из NLRO в SRO может сдвигаться до значения $p_{0}^{\mathrm{N}}(\gamma=1.0) \approx 0.25$, что хорошо совпадает с кластерными расчетами [35]. Это позволяет считать, что при подходе к точке фазового перехода параметр $\gamma$ увеличивается с увеличением фрустрации (имеется в виду, что при нулевой фрустрации $\gamma$ мало или равно нулю).

Вообще говоря, считается, что при $T=0$ в модели $J_{1}$ затухание при $\mathbf{q}=\mathbf{Q}$ отсутствует. Однако если учесть, что при вычислении $M(\mathbf{q}, \omega)$ в соответствующие неприводимые одномагнонные функции Грина следует подставлять самосогласованное решение, то это утверждение является неочевидным. В предположении, что при $T=0, p=0$ существует малое, но конечное затухание, можно в рамках приближения (15) удовлетворить условию, что $c_{0}^{\mathrm{N}}$ и средняя энергия одновременно с высокой точностью совпадают с данными, полученными методом Монте-Карло (см., например, обсуждения и ссылки в работе [8]).

Наиболее разительным является изменение зависимости спиновой щели при включении затухания. На рис. 4 представлена зависимость спиновой щели от $T$ в АФМ-точке $\mathbf{Q}$ для различных значений $\gamma$ и $p$. Отметим прежде всего, что величину щели корректно определять не по значению самосогласованного спектра $\Delta_{\mathbf{Q}}=\omega_{\mathbf{q}=\mathbf{Q}}$, а по положению максимума пика $\chi^{\prime \prime}(\mathbf{Q}, \omega)$ по $\omega$, т. е. $\tilde{\Delta}_{\mathbf{Q}}=\max \chi^{\prime \prime}(\mathbf{Q}, \omega)$. Вид $G^{\prime \prime}(\mathbf{q}, \omega)(15)$ приводит к тому, что всегда $\omega_{\mathbf{q}}>\widetilde{\omega}_{\mathbf{q}}=\max \chi^{\prime \prime}(\mathbf{q}, \omega)$. Сравним значения щели $\tilde{\Delta}_{\mathbf{Q}}$ при $p=0.2, T=0.1, \gamma=0.3$ на рис. 4 со среднеполевым значением $\omega_{\mathbf{Q}}$, представленным для тех же значений $p$ и $T$ на рис. 3. Легко видеть, что $\tilde{\Delta}_{\mathbf{Q}} \lesssim 0.5 \omega_{\mathbf{Q}}$.

Проведенные рассмотрения показывают, что рост величины затухания приводит к уменьшению спиновой щели в области SRO-фазы и к увеличению конденсата в LRO-фазах, что является нетривиальным, с нашей точки зрения. Таким образом, рост $\gamma$ способствует усилению LRO. Продемонстрируем на примере LRO-фазы при $T=0$, что это обстоятельство является следствием существования правила сумм для $G(\mathbf{q}, \omega)$, т. е. констрейнта $\left\langle S_{\mathbf{n}}^{\alpha} S_{\mathbf{n}}^{\alpha}\right\rangle=1 / 4$. Для функции Грина $G(\mathbf{q}, \omega)(15)$ констрейнт имеет вид

$$
\begin{gathered}
\frac{1}{4}=I(\gamma)+c_{0}(\gamma), \quad I(\gamma)=\frac{1}{4 \pi^{3}} \iint d \mathbf{q} J\left(\omega_{\mathbf{q}}, \gamma\right) \\
J\left(\omega_{\mathbf{q}}, \gamma\right)=\int_{0}^{+\infty} d \omega \frac{F_{\mathbf{q}}(\gamma) \omega \gamma}{\left(\omega^{2}-\omega_{\mathbf{q}}^{2}(\gamma)\right)^{2}+\omega^{2} \gamma^{2}}
\end{gathered}
$$




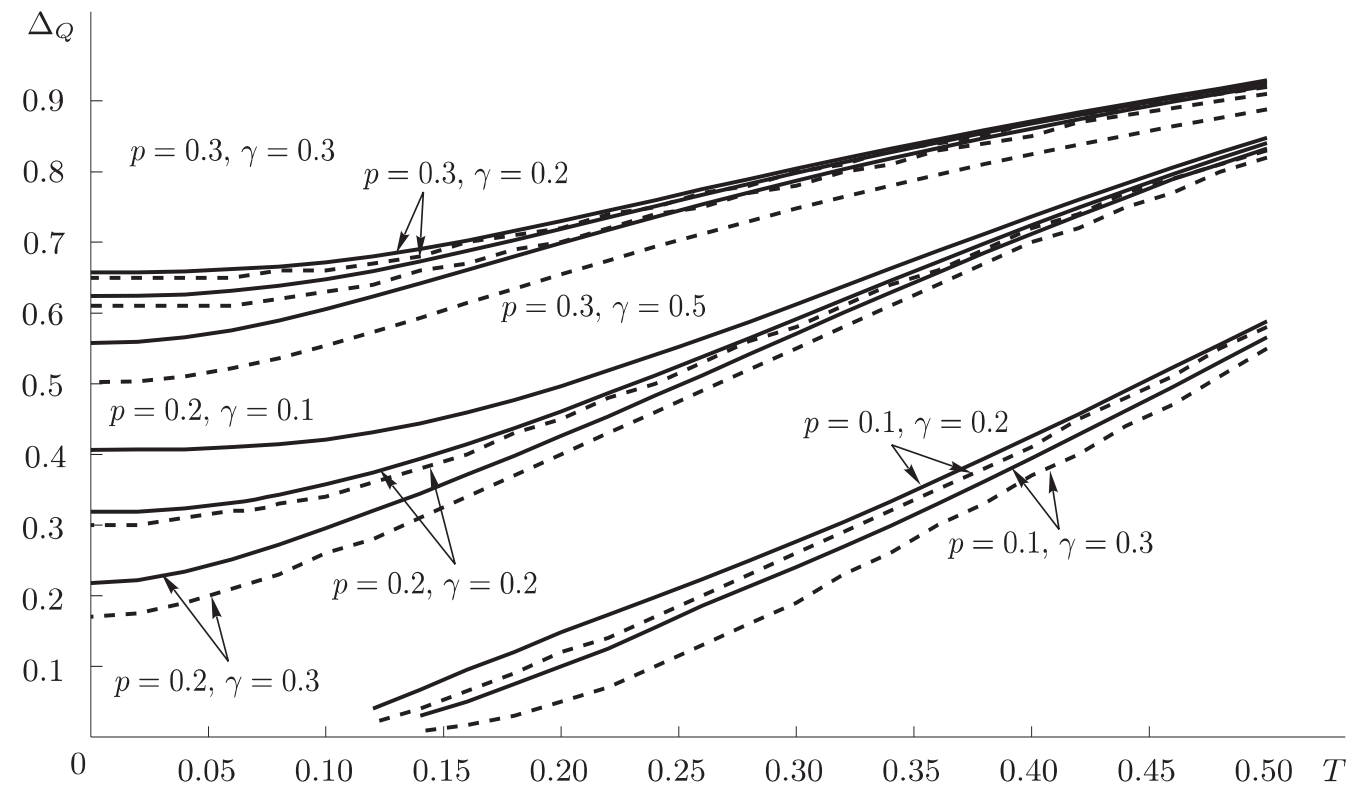

Рис. 4. Зависимость спиновой щели $\Delta_{\mathbf{Q}}=\omega_{\mathbf{q}=\mathbf{Q}}(\mathbf{Q}=(\pi, \pi))$ от температуры при различных значениях $\gamma$ и $p=0.1,0.2,0.3$ (сплошные линии). Штриховые линии - то же для щели $\tilde{\Delta}_{\mathbf{Q}}$, определенной по положению максимума $\chi^{\prime \prime}(\mathbf{Q}, \omega)$ (для последних кривых точность вычислений 0.01 ). При $p=0.2, \gamma=0.1$ сплошная и штриховая линии в масштабе рисунка практически сливаются.

Покажем, что $d c_{0}(\gamma) / d \gamma=-d I(\gamma) / d \gamma>0$. Для этого представим $d I(\gamma) / d \gamma$ в виде

$$
\begin{gathered}
\frac{d I(\gamma)}{d \gamma}=I_{\gamma}^{\prime}=I_{1}^{\prime}+I_{2}^{\prime}, \quad I_{1}^{\prime}=\left.\frac{d I\left(\gamma_{1} ; \gamma\right)}{d \gamma_{1}}\right|_{\gamma_{1}=\gamma}, \quad I_{1}^{\prime}=\left.\frac{d I\left(\gamma ; \gamma_{2}\right)}{d \gamma_{2}}\right|_{\gamma_{2}=\gamma}, \\
I\left(\gamma_{1} ; \gamma_{2}\right)=\frac{1}{4 \pi^{3}} \int_{0}^{+\infty} d \omega \int_{\mathbf{q}} \frac{F_{\mathbf{q}}\left(\gamma_{2}\right) \omega \gamma_{1}}{\left(\omega^{2}-\omega_{\mathbf{q}}^{2}\left(\gamma_{2}\right)\right)^{2}+\omega^{2} \gamma_{1}^{2}} d \mathbf{q}
\end{gathered}
$$

где $F_{\mathbf{q}}\left(\gamma_{2}\right)$ и $\omega_{\mathbf{q}}\left(\gamma_{2}\right)$ отвечают самосогласованным величинам, вычисленным при затухании $\gamma_{2}$. Производная $I_{1}^{\prime}(\gamma)$ соответствует дифференцированию по явной зависимости $I(\gamma)$, а $I_{2}^{\prime}(\gamma)$ - дифференцированию по неявной зависимости $I(\gamma)$ от $\gamma$ через $\omega_{\mathbf{q}}(\gamma)$ и $F_{\mathbf{q}}(\gamma)$. Прямые расчеты показывают, что в LRO-фазах $\left|I_{2}^{\prime} / I_{1}^{\prime}\right| \lesssim 0.2$. Это означает, что для нахождения $I^{\prime}$ достаточно ограничиться первым членом $I_{1}^{\prime}$,

$$
I_{1}^{\prime}=\frac{1}{4 \pi^{3}} \int_{0}^{+\infty} d \omega \int_{\mathbf{q}} d \mathbf{q} F_{\mathbf{q}} \omega \frac{\left(\omega^{2}-\omega_{\mathbf{q}}^{2}\right)^{2}-\omega^{2} \gamma^{2}}{\left[\left(\omega^{2}-\omega_{\mathbf{q}}^{2}\right)^{2}+\omega^{2} \gamma^{2}\right]^{2}}
$$

Для того чтобы убедиться, что $I^{\prime}<0$ при любых значениях $\gamma$, достаточно рассмотреть выражение для подынтегральной функции $J\left(\omega_{\mathbf{q}}, \gamma\right)$ в $(16)$ и ее производной по $\gamma$ при фиксированных значениях $F_{\mathbf{q}}, \omega_{\mathbf{q}}^{2}$.

В выражении для $J\left(\omega_{\mathbf{q}}, \gamma\right)$ интегрирование по $\omega$ проводится явно, и анализ показывает, что при любых $\omega_{\mathbf{q}}$ и $\gamma$ выполняется $J_{\gamma}^{\prime}\left(\omega_{\mathbf{q}}, \gamma\right)<0$, а следовательно, 
$d c_{0}(\gamma) / d \gamma>0$. Качественно это можно понять, если учесть, что при возрастании $\gamma$ вклад (16) от интегрирования по $d \omega$ обрезается нижним пределом. Если бы, например, под интегралом стоял лоренциан $Г /\left[\left(\omega^{2}-\omega_{\mathbf{q}}^{2}\right)^{2}+\Gamma^{2}\right]$ и интегрировался бы по $\omega$ от $-\infty$ до $+\infty$, то ответ не зависел бы от $\Gamma$; при интегрировании от нуля до бесконечности очевидно, что интеграл уменьшается с ростом Г. Конечно, в нашем случае подынтегральное выражение в $J\left(\omega_{\mathbf{q}}, \gamma\right)$ отличается от лоренциана, однако качественно результат остается тем же: рост $\gamma$ уменьшает величину $J\left(\omega_{\mathbf{q}}, \gamma\right)$.

Увеличение конденсата в LRO-фазе при росте $\gamma$ в случае $T=0$ качественно соответствует уменьшению спиновой щели при $T>0$. На рис. 4 (зависимость спиновой щели $\Delta_{\mathbf{Q}}=\omega_{\mathbf{q}=\mathbf{Q}}, \mathbf{Q}=(\pi, \pi)$, от температуры при различных значениях $\gamma$ и $\left.p\right)$ видно, что при фиксированном значении фрустрации щель монотонно убывает с ростом $\gamma$ в широкой области температур. На том же рисунке приведено рассчитанное поведение спиновой щели $\tilde{\Delta}_{\mathbf{Q}}$, определенное по максимуму функции $\chi^{\prime \prime}(\mathbf{Q}, \omega)$, которую измеряют в нейтронных экспериментах. Отметим вновь, что благодаря отличию $\chi^{\prime \prime}(\mathbf{q}, \omega)$ от лоренциана всегда $\tilde{\Delta}_{\mathbf{q}}<\Delta_{\mathbf{q}}$. Как видно из рис. $4, \tilde{\Delta}_{\mathbf{Q}}$ также уменьшается при увеличении $\gamma$, что отвечает усилению LRO, т. е. увеличению корреляционной длины. Это обстоятельство связано с констрейнтом и существенно отличает систему спиновых флуктуаций от чисто бозевской системы, например фононов. Для фононной подсистемы условие, подобное спиновому констрейнту, соответствовало бы требованию, чтобы среднее от квадрата смещения узла оставалось постоянным и не зависело от температуры. Такого условия у фононной подсистемы нет, а правило сумм для фононной функции Грина не имеет ничего общего с правилом сумм для спиновых возбуждений. Что касается обращения в нуль спектра в некоторой отличной от нуля точке зоны Бриллюэна, то такая ситуация для фононной системы может реализовываться вблизи структурного фазового перехода.

3.2. Скейлинговое поведение спиновой восприимчивости. Важнейшая величина, получаемая из нейтронных экспериментов в купратах [6], [36]-[40], - это локальная восприимчивость $\chi_{2 \mathrm{D}}(\omega)$, которая определяется как мнимая часть спиновой восприимчивости, проинтегрированная по квазиимпульсу:

$$
\chi_{2 \mathrm{D}}(\omega, T)=\int d \mathbf{q} \chi^{\prime \prime}(\mathbf{q}, \omega, T) .
$$

В области нулевого и малого допирования величина $\chi_{2 \mathrm{D}}(\omega)$ в широком диапазоне частот практически постоянна (см. рис. 5), она начинает расти при высоких частотах для $\omega \gtrsim 150 \mathrm{MэB} \mathrm{[41],} \mathrm{[42].} \mathrm{Такое} \mathrm{поведение} \mathrm{проинтегрированной} \mathrm{восприимчивости}$ слабо зависит от температуры. При низких частотах $\chi_{2 \mathrm{D}}(\omega)$ меняется для частот, меньших щели $\Delta$ в спектре спиновых возбуждений (последнее не видно на рис. 5 из-за ограниченного числа частотных точек), и ее поведение существенно зависит от температуры $T$.

Один из наиболее ярких экспериментальных результатов - это скейлинг $\chi_{2 \mathrm{D}}$ : зависимость $\chi_{2 \mathrm{D}}$ от $\omega$ и $T$ хорошо (а при малом допировании - практически идеально) описывается эмпирическим скейлинговым законом [1], [36], [43]-[46]

$$
\frac{\chi_{2 \mathrm{D}}(\omega, T)}{\chi_{2 \mathrm{D}}(\omega, T \rightarrow 0)}=f\left(\frac{\omega}{T}\right),
$$


где скейлинговая функция чаще всего аппроксимируется выражением $f(x)=$ $(2 / \pi) \operatorname{arctg}\left(A x+B x^{3}\right), A$ и $B$ зависят от допирования, причем при нулевом и малом допировании $B \gg A \sim 1$, а с ростом допирования коэффициент $B$ резко убывает.

В диэлектрическом пределе экспериментальный спектр спиновых возбуждений $\omega_{\mathbf{q}}$ [47] качественно похож на спектр двумерного АФМ в приближении линейных спиновых волн. При увеличении концентрации дырок поведение функции $\chi_{2 \mathrm{D}}(\omega)$ радикально меняется. Ее пик, который при малом допировании приходится на область максимума спектра $\omega \gtrsim 200 \mathrm{MэВ,} \mathrm{с} \mathrm{ростом} \mathrm{допирования} \mathrm{при} \mathrm{низких} T$ смещается вниз, опускаясь при оптимальном допировании до $\omega \sim 35 \mathrm{MэB} \mathrm{(см.} \mathrm{рис.} 5$ ).

Покажем, что в рамках СССП (15) оказывается возможным аналитически исследовать скейлинг восприимчивости $\chi_{2 \mathrm{D}}(\omega)$.

На рис. 6 представлена эволюция с ростом фрустрации восприимчивости $\chi_{2 \mathrm{D}}(\omega)$, полученной в среднеполевом приближении. При малых фрустрациях величина $\chi_{2 \mathrm{D}}(\omega)$ в среднеполевом приближении в области малых частот представляет собой ступеньку $\left(\chi_{2 \mathrm{D}}(\omega) \sim \theta\left(\omega-\Delta_{\mathbf{Q}}\right)\right)$, что, очевидно, делает невозможным объяснение скейлинга восприимчивости ${ }^{1)}$.

Представленные на рис. 7,8 результаты получены при значении параметра фрустрации $p=0.1$, что отвечает недодопированной ситуации в купратах, когда скейлинг выполняется лучше всего.

Для $p=0.1$ при низких температурах $(T<0.1 J)$ корреляторы стабилизированы и почти не зависят от температуры, однако щель остается чувствительной к небольшим их изменениям. Мы положили $T=0.01$ для знаменателя скейлингового выражения (18), при этом щель оказывается равной $\Delta=0.005$ (при $\gamma=0.045$ ). Этим значениям параметров отвечает корреляционная длина (мы определяли ее по полуширине пика $C_{\mathbf{q}}$ на полувысоте, как это обычно делается при обработке эксперимента) $\xi>500 \AA$ (в этом разделе для постоянной решетки $\mathrm{CuO}_{2}$ принято $a=5.354 \AA)$.

Для $T>0.01$ при каждом значении температуры вычислялись восприимчивость, спектр и скейлинговая функция. Значение затухания $\gamma$ подбиралось таким, чтобы наилучшим образом воспроизвести скейлинг (18) (напомним, что в рамках используемой процедуры при изменении $\gamma$ корреляторы, спектр и щель изменяются самосогласованным образом). Оказалось, что скейлинг хорошо воспроизводится, если затухание зависит от температуры линейно с близким к единице коэффициентом, т. е. при $\gamma=T$. Ниже будет показано аналитически, что, наоборот, предположение $\gamma \sim T$ является достаточным условием выполнения скейлинга.

Полученные численно в предположении $\gamma=T$ спектры спиновых возбуждений представлены на рис. 7. Из рисунка ясно, что сильнее всего спектры зависят от температуры в окрестности АФМ-точки Q. Видно также, что при достаточно низких температурах спектр линеен по $\tilde{q}=|\mathbf{Q}-\mathbf{q}|$ (а область квадратичной зависимости почти неразличима).

Скейлинговые кривые (полученные при $\gamma=T$ ) для нескольких температур $0.05 \leqslant$ $T \leqslant 0.4$ представлены на рис. 8. Там же представлены функции $(2 / \pi) \operatorname{arctg}(1.3 x)$ и $1 /\left(n_{B}(x)+1\right)$, которыми разумно описываются экспериментальные данные при не слишком большом допировании (эти две зависимости используются для аппроксимации экспериментальной скейлинговой функции при малом допировании [45]). На

1) В частности, ниже на рис. 9 продемонстрировано кривыми при $\gamma=0.1 J, T=0.4 J$ и $\gamma=0.1 J$, $T=0.1 J$, что при $\gamma$, не зависящем от $T$ (среднеполевой случай $-\gamma=0$ при любых $T$ ), невозможно описать скейлинговое поведение. 


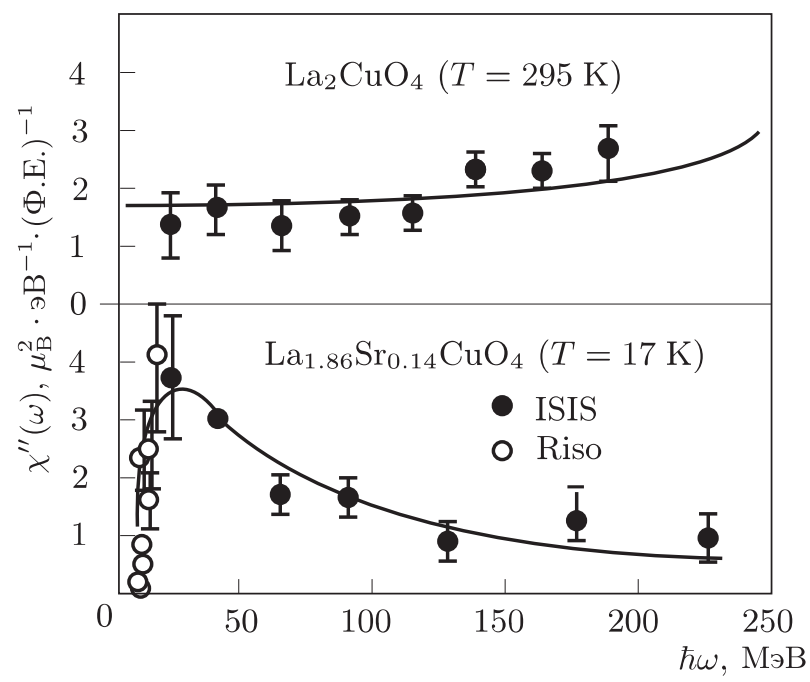

Рис. 5. Характерное изменение экспериментальной проинтегрированной восприимчивости $\chi_{2 \mathrm{D}}(\omega)$ с ростом допирования [41]. Верхняя кривая получена для диэлектрика $\mathrm{La}_{2} \mathrm{CuO}_{4}$, нижняя - для допирования, близкого к оптимальному для $\mathrm{La}_{1.86} \mathrm{Sr}_{0.14} \mathrm{CuO}_{4}\left(T_{\mathrm{c}}=35 \mathrm{~K}\right)$. В скобках указана температура измерения. Заполненные и пустые кружки соответствуют разным нейтронным источникам.

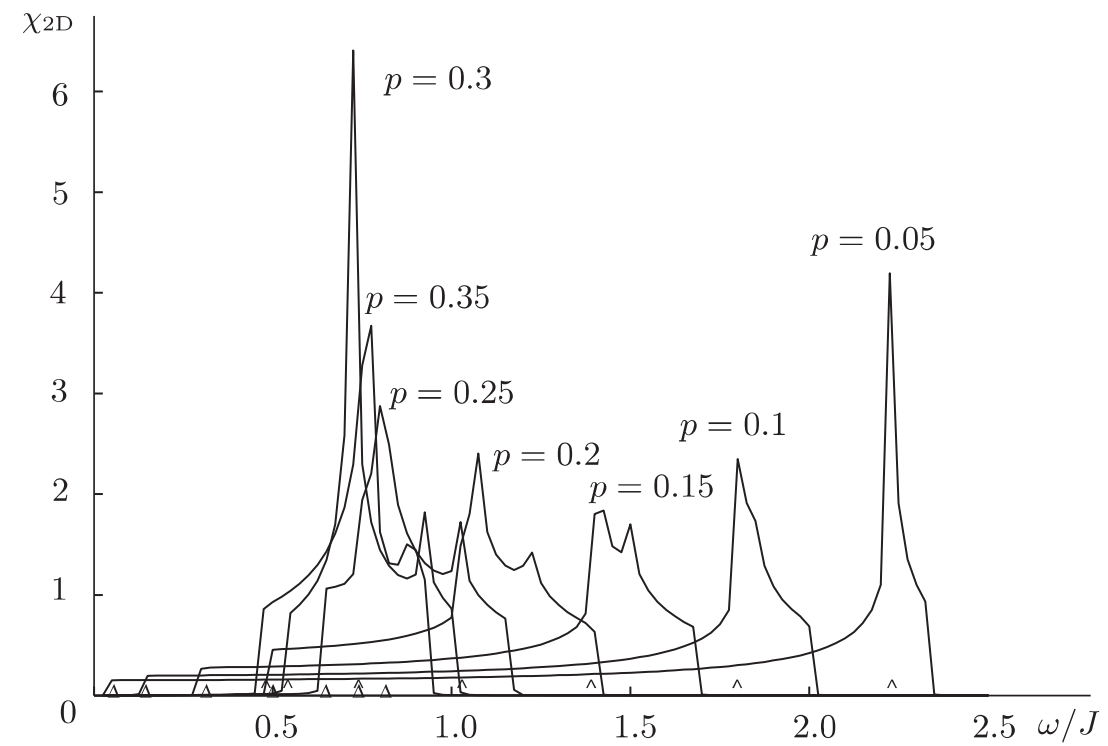

Рис. 6. Изменение проинтегрированной восприимчивости $\chi_{2 \mathrm{D}}(\omega)$ в среднеполевом приближении с ростом фрустрации. Знаком $\Delta$ показано положение щели в точке $\mathbf{Q}=(\pi, \pi)$ при данной фрустрации (с ростом параметра $p$ эта щель монотонно возрастает), знаком ^ - щель в точках $\mathbf{X}=(0, \pi)$ (с ростом $p$ она монотонно убывает). 


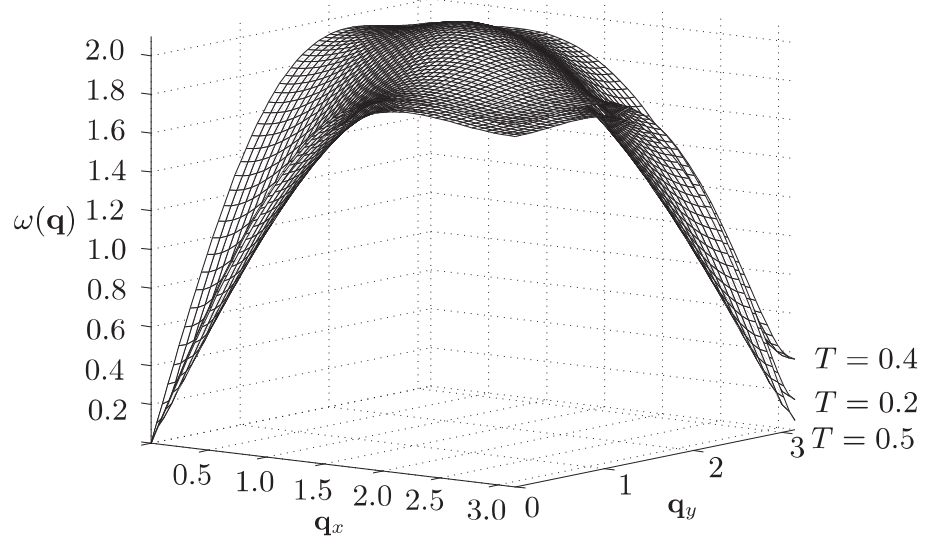

Рис. 7. Самосогласованный спектр спиновых возбуждений $\omega_{\mathbf{q}}$ для значения параметра фрустрации $p=0.1$ и температур $T=0.05 J, 0.2 J, 0.4 J$ (показана четверть зоны Бриллюэна).

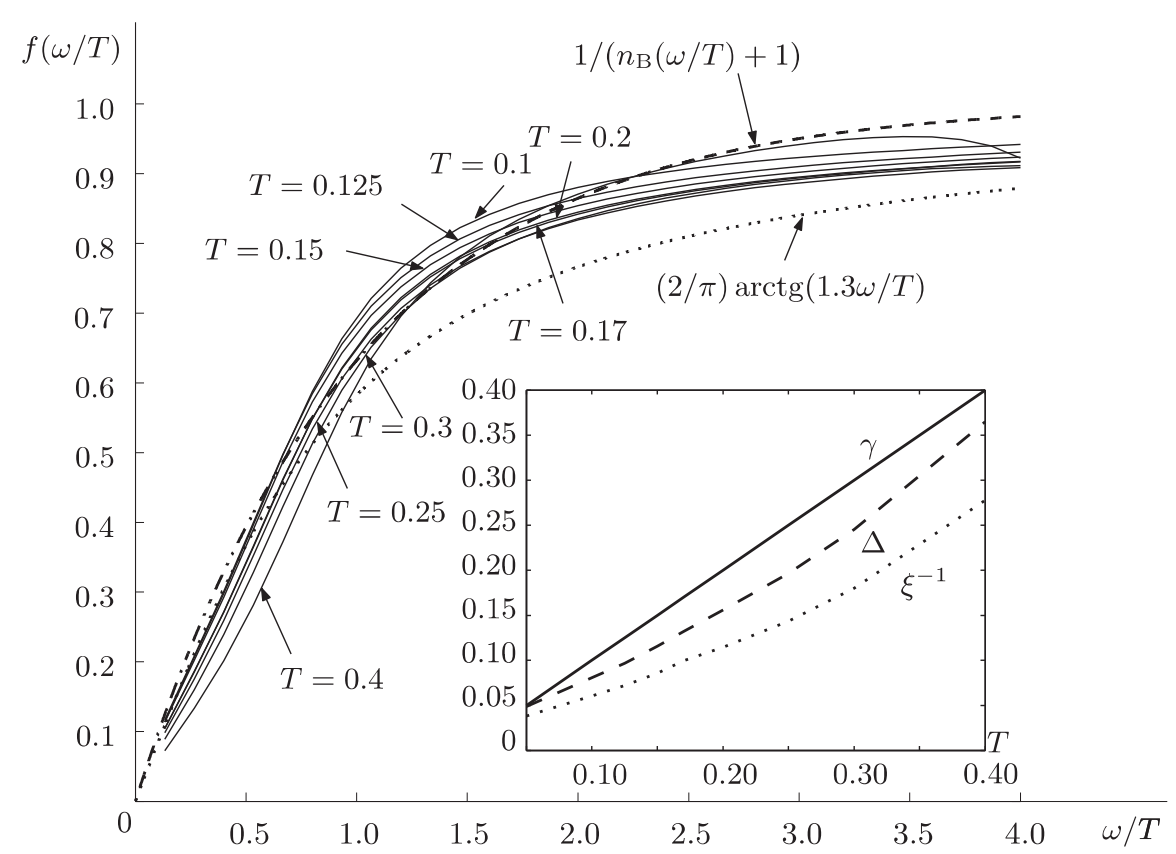

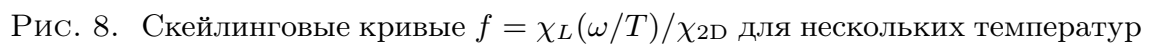
$0.1 J \leqslant T \leqslant 0.4 J$ как функции $\omega / T$. Параметр фрустрации $p=0.1$. Пунктиром представлена кривая $(2 / \pi) \operatorname{arctg}(1.3 \omega / T)$, штрихпунктирная линия $(m(\omega / T)+1)^{-1}$, где $m-$ бозе-функция. 


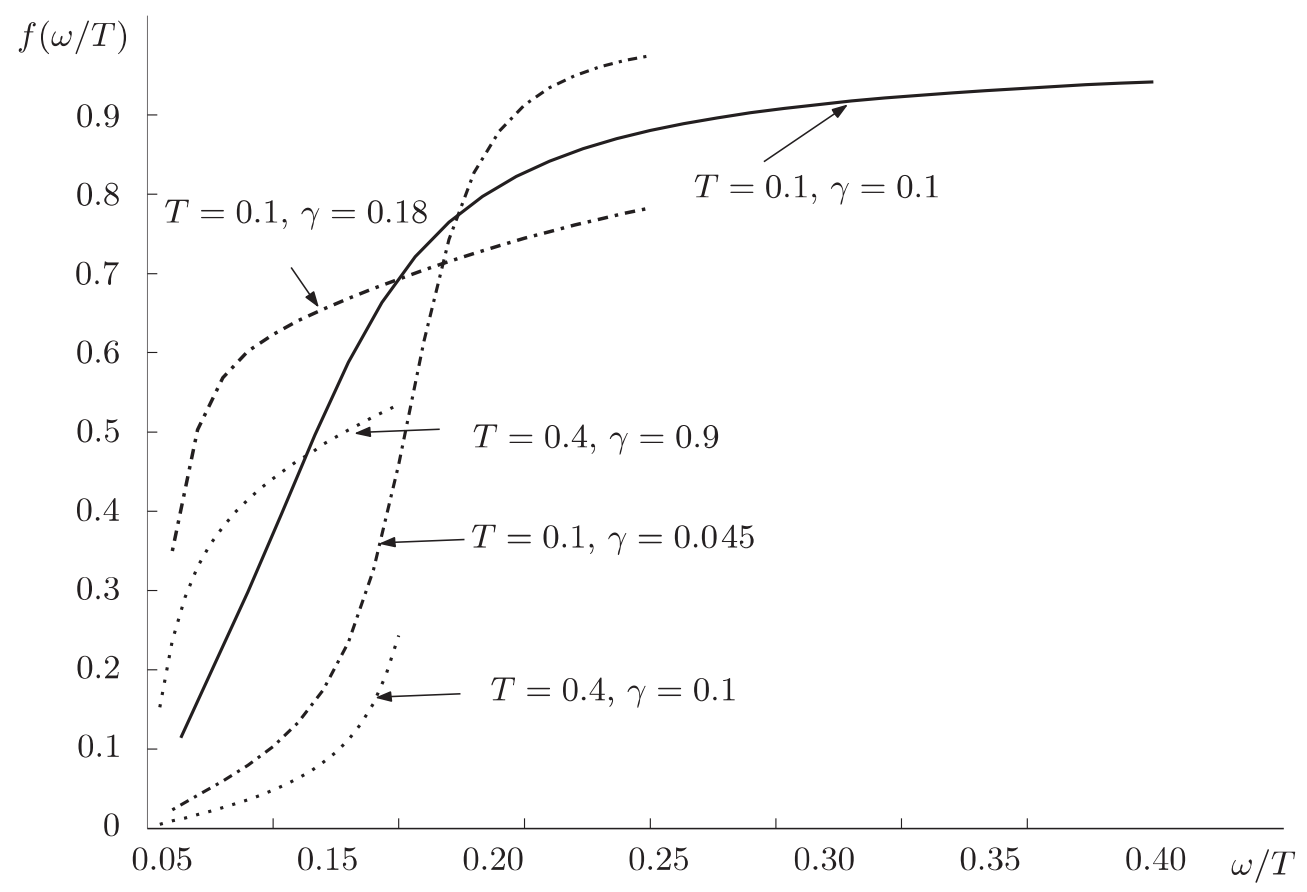

Рис. 9. Отклонения от оптимального скейлинга при $\gamma \neq T$. Сплошная кривая - линия оптимального скейлинга.

вставке показаны температурные зависимости затухания $\gamma$ и щели $\Delta$. Как видно, щель $\Delta$ также ведет себя линейно с температурой. Там же приведена температурная зависимость обратной корреляционной длины $\xi^{-1}$. Она хорошо согласуется с экспериментальными данными для $\mathrm{La}_{1.97} \mathrm{Sr}_{0.03} \mathrm{CuO}_{4}$ [43].

На рис. 9 продемонстрировано, что при отклонении величины затухания $\gamma$ от линейной зависимости $\gamma=T$ скейлинговый закон существенно нарушается.

Итак, при $p=0.1$ численные результаты демонстрируют скейлинг $\chi_{2 \mathrm{D}}(\omega, T)$, если $\gamma=T$, и приводят к разумному описанию поведения корреляционной длины. Упомянем, не приводя соответствующих графиков, что при $p<0.1$ в предположении $\gamma=\alpha T$ (где величина $\alpha$ зависит от фрустрации и не обязательно равна единице) скейлинг численно воспроизводится с еще большей точностью.

Наконец, покажем, что при $p \lesssim 0.3$ вопрос о скейлинговой кривой может быть прояснен аналитически. Как ясно из выражения для $\operatorname{Im} \chi(\mathbf{q}, \omega)$, основной вклад в $\chi_{2 \mathrm{D}}(\omega)$ при не слишком больших частотах дает область вблизи точки $\mathbf{Q}: \tilde{q}=$ $|\mathbf{q}-\mathbf{Q}| \leqslant \tilde{q}_{0}$, где $c \tilde{q}_{0} \sim J$ - наибольший энергетический параметр задачи (вклад от окрестности $\mathbf{q}=0$ подавляется числителем $\left.F_{\mathbf{q}}\right)$. Тогда разложение спектра $\omega^{2}(q) \approx$ $\Delta^{2}+c^{2} \tilde{q}^{2}\left(\Delta \equiv \Delta_{\mathbf{Q}}\right)$ и простое интегрирование $\chi_{2 \mathrm{D}}(\omega, T)$ дают для $\omega<\omega_{0}=c \tilde{q}_{0}$

$$
\begin{aligned}
\chi_{2 \mathrm{D}}(\omega) & =\frac{\bar{F}_{\mathbf{q}}}{4 \pi c^{2}}\left\{\operatorname{arctg}\left(\frac{c^{2} \tilde{q}_{0}^{2}+\Delta^{2}-\omega^{2}}{\gamma \omega}\right)-\operatorname{arctg}\left(\frac{\Delta^{2}-\omega^{2}}{\gamma \omega}\right)\right\}= \\
& =\frac{\bar{F}_{\mathbf{q}}}{4 \pi c^{2}} \begin{cases}\Phi(\omega, \Delta, \gamma), & \theta<1, \\
\pi+\Phi(\omega, \Delta, \gamma), & \theta>1,\end{cases}
\end{aligned}
$$




$$
\begin{aligned}
\Phi(\omega, \Delta, \gamma) & =\operatorname{arctg}\left\{\frac{c^{2} \tilde{q}_{0}^{2} \gamma \omega}{\gamma^{2} \omega^{2}+\left(c^{2} \tilde{q}_{0}^{2}+\Delta^{2}-\omega^{2}\right)\left(\Delta^{2}-\omega^{2}\right)}\right\} \\
\theta & =\frac{\left(c^{2} \tilde{q}_{0}^{2}+\Delta^{2}-\omega^{2}\right)\left(\omega^{2}-\Delta^{2}\right)}{\gamma^{2} \omega^{2}}
\end{aligned}
$$

где $\bar{F}_{\mathbf{q}}-$ среднее от медленно меняющейся в рассматриваемой области функции $F_{\mathbf{q}}$.

Из эксперимента известно, что скейлинговый знаменатель $\chi_{2 \mathrm{D}}(\omega, T \rightarrow 0)$ постоянен в широком диапазоне частот. Это же видно из соотношения (19), так как $\Delta, \gamma \rightarrow 0$ при $T \rightarrow 0$, тогда $\chi_{2 \mathrm{D}}(\omega, T \rightarrow 0)=\bar{F}_{\mathbf{q}} / 4 c^{2}$. Таким образом, скейлинг фактически управляется числителем $\chi_{2 \mathrm{D}}(\omega, T)$, т. е. функцией $\Phi(\omega, \Delta, \gamma)$, которая при $\Delta^{2}, \omega^{2}, \gamma^{2} \ll c^{2} \tilde{q}_{0}^{2}$ принимает вид

$$
\Phi(\omega, \Delta, \gamma) \approx \operatorname{arctg}\left(\frac{\gamma \omega}{\Delta^{2}-\omega^{2}}\right)=\operatorname{arctg}\left(\frac{(\gamma / T) \omega / T}{(\Delta / T)^{2}-(\omega / T)^{2}}\right) .
$$

Выражение (19) доказывает, что линейное поведение щели и затухания по температуре $\gamma=\alpha T$ и $\Delta=\beta T$ является необходимым условием скейлинга (подчеркнем, что при малых $p$ линейность щели по температуре хорошо выполняется при линейности $\gamma$; см. ниже рис. 12).

В рассматриваемом пределе переключение режимов $(19),(21)$ определяется $\Theta$-функцией $\Theta(\theta) \simeq \Theta\left(\omega^{2}-\Delta^{2}\right)=\Theta\left((\omega / T)^{2}-\beta^{2}\right)$. Тогда скейлинговая функция приобретает вид

$$
\tilde{f}\left(\frac{\omega}{T}\right)=\pi \Theta\left(\left(\frac{\omega}{T}\right)^{2}-\beta^{2}\right)+\operatorname{arctg}\left(\frac{\alpha \omega / T}{\beta^{2}-(\omega / T)^{2}}\right) .
$$

Таким образом, в противоположность экспериментальным подгонкам с помощью простого арктангенса или арктангенса от кубического полинома, здесь получается арктангенс "с переключателем" (переключение происходит при $\omega=\Delta$ ), содержащий к тому же микроскопическую информацию о $\Delta$ и $\gamma$.

Итак, в рассмотренном аналитическом приближении величина $\chi_{2 \mathrm{D}}(\omega)$ (а следовательно, и скейлинговая функция $\tilde{f}(\omega / T))$ при $\omega \lesssim J$ определяется выражением (23) и представляет собой ступеньку, обращающуюся в нуль при $\omega \sim \Delta$ и размытую на ширину $\sim \gamma$. Однако измерения при гелиевых температурах в слабодопированных купратах демонстрируют отклонение от такого поведения: на самых низких частотах $\chi_{2 \mathrm{D}}(\omega)$ начинает расти и лишь затем обращается в нуль [43]-[45].

Не приводя соответствующих выкладок, отметим, что такое поведение также можно продемонстрировать аналитически, если считать, что в очень узкой окрестности точки $\mathbf{Q}$ спектр уплощается по отношению к использованному выше дираковскому виду $\left(\widetilde{\omega}^{2}(q) \approx \Delta^{2}+c^{2} \tilde{q}^{2}\right)$, т. е. для $\omega<\widetilde{\omega}_{0} \ll \omega_{0}$ спектр имеет вид $\widetilde{\omega}^{2}(q) \approx \Delta^{2}+\mu^{2} \tilde{q}^{4}$.

Итак, мы показали, что введение затухания $\gamma$ и предположение о его линейности по температуре позволяют воспроизвести экспериментальное скейлинговое поведение без привлечения феноменологических подгоночных формул.

Представляется, что наш подход выгодно отличается от метода линейных спиновых волн, который также дает явный вид для спектра возбуждений [16], [38], [48], [49], однако справедлив только в диэлектрическом пределе, имеющиеся его реализации не учитывают затухания, применимы лишь при $T=0$ и не являются самосогласованными. 
q, единицы обратной решетки

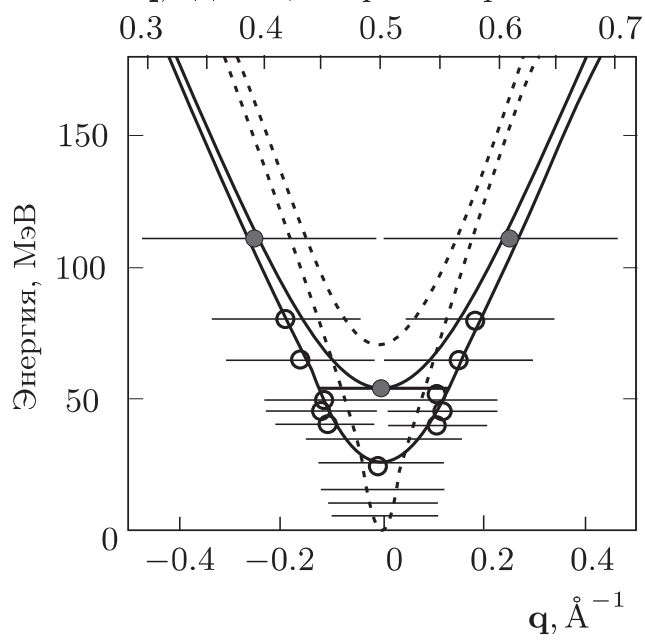

a

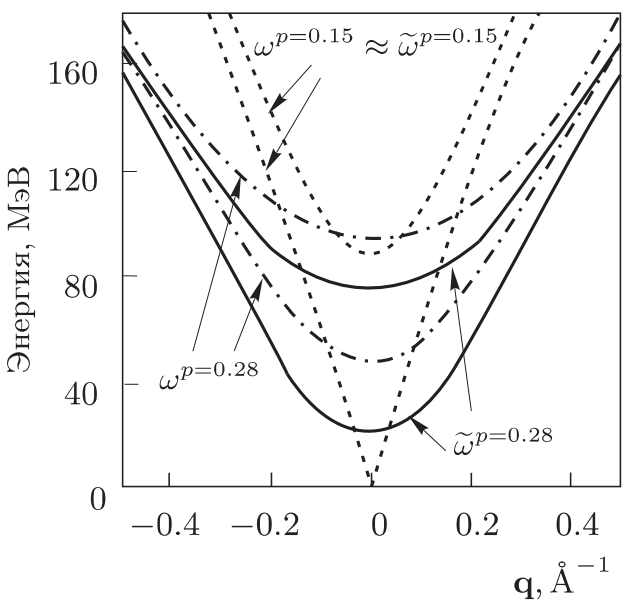

б

Рис. 10. Спектр акустических и оптических мод относительно точки $\mathbf{Q}=(\pi ; \pi)$ для двух плоскостей $J_{1}-J_{2}$ с межплоскостным АФМ-обменом $J_{\perp}=$ 12 МэВ. (а) - Данные нейтронных экспериментов [37]. Штриховые линии отвечают спектрам $\omega_{\text {ac,opt }}^{x=0.15}(\mathbf{q})$ диэлектрика $\mathrm{YBa}_{2} \mathrm{Cu}_{3} \mathrm{O}_{6.15}$ при $T=296 \mathrm{~K}$ [42]. Пустые и заполненные кружки отвечают соответственно $\omega_{\mathrm{ac}}^{x=0.5}(\mathbf{q})$ и $\omega_{\mathrm{opt}}^{x=0.5}(\mathbf{q})$ для допированного $\mathrm{YBa}_{2} \mathrm{Cu}_{3} \mathrm{O}_{6.5}$ при $T=5 \mathrm{~K}$, сплошные линии - квадратичная подгонка $\omega^{2}=\omega_{0}^{2}+c^{2} q^{2}$. (б) - Вычисленные спектры для двух значений фрустрации $p=0.15\left(T_{p=0.15}=0.25 J_{1}, \gamma_{p=0.15} \approx 0.1 J_{1}\right)$ и $p=0.28$ $\left(T_{p=0.28}=0.07 J_{1}, \gamma_{p=0.28}=J_{1}\right)$ при фиксированных значениях $J_{1}=120 \mathrm{MэB}$, $J_{\perp}=12$ МэВ. Штриховые линии отвечают спектрам $\widetilde{\omega}_{\mathrm{ac}, \mathrm{opt}}^{p=0.15}(\mathbf{q})$ при $p=0.15$ (соответствуют штриховым линиям на рисунке а). В случае $p=0.28$ : штрихпунктирные линии - $\omega_{\mathrm{ac}, \mathrm{opt}}^{p=0.28}(\mathbf{q})$; сплошные линии - спектры $\widetilde{\omega}_{\mathrm{ac}, \mathrm{opt}}^{p=0.28}(\mathbf{q})$, определяемые по положениям пиков у $\chi_{\mathrm{ac}, \mathrm{opt}}^{\prime \prime}(\mathbf{q}, \omega)$ (ср. со сплошными линиями на рисунке а).

\section{3. Восприимчивость двухплоскостных купратов и несоизмеримые} $\chi(\mathbf{q}, \omega)$-пики. Остановимся кратко на результатах, которые демонстрируют, во-первых, что учет затухания позволяет воспроизвести экспериментальные результаты для акустических и оптических мод $\chi_{\mathrm{ac}, \mathrm{opt}}^{\prime \prime}(\mathbf{q}, \omega)$ в двухплоскостных $\mathrm{YBa}_{2} \mathrm{Cu}_{3} \mathrm{O}_{6+x}$ как при малом, так и при большом допировании [50] и, во-вторых, что разумное усложнение функции $M(\mathbf{q}, \omega)$ в (14) позволяет воспроизвести несоизмеримые пики $\chi^{\prime \prime}(\mathbf{q}, \omega)$ при переходе допированного АФМ в сверхпроводящее состояние [32].

В двухплоскостном случае мы принимаем, что внутри каждой плоскости реализуется модель $J_{1}-J_{2}$, а межплоскостному АФМ-взаимодействию между ближайшими узлами, относящимися к разным плоскостям, отвечает константа обмена $J_{\perp}$. Введение константы $J_{\perp}$ приводит к нетривиальному расщеплению одноплоскостного решения $\chi(\mathbf{q}, \omega)$. Расщепление описывает две ветви оптической $\chi_{\text {opt }}(\mathbf{q}, \omega)$ и акустической $\chi_{\text {ac }}(\mathbf{q}, \omega)$ восприимчивости. Для реалистических значений параметров $J_{1}=120 \mathrm{MэB}, J_{\perp}=12$ МэВ результаты для спектров $\omega_{\text {ac, орt }}(\mathbf{q})$ и их сравнение с экс- 


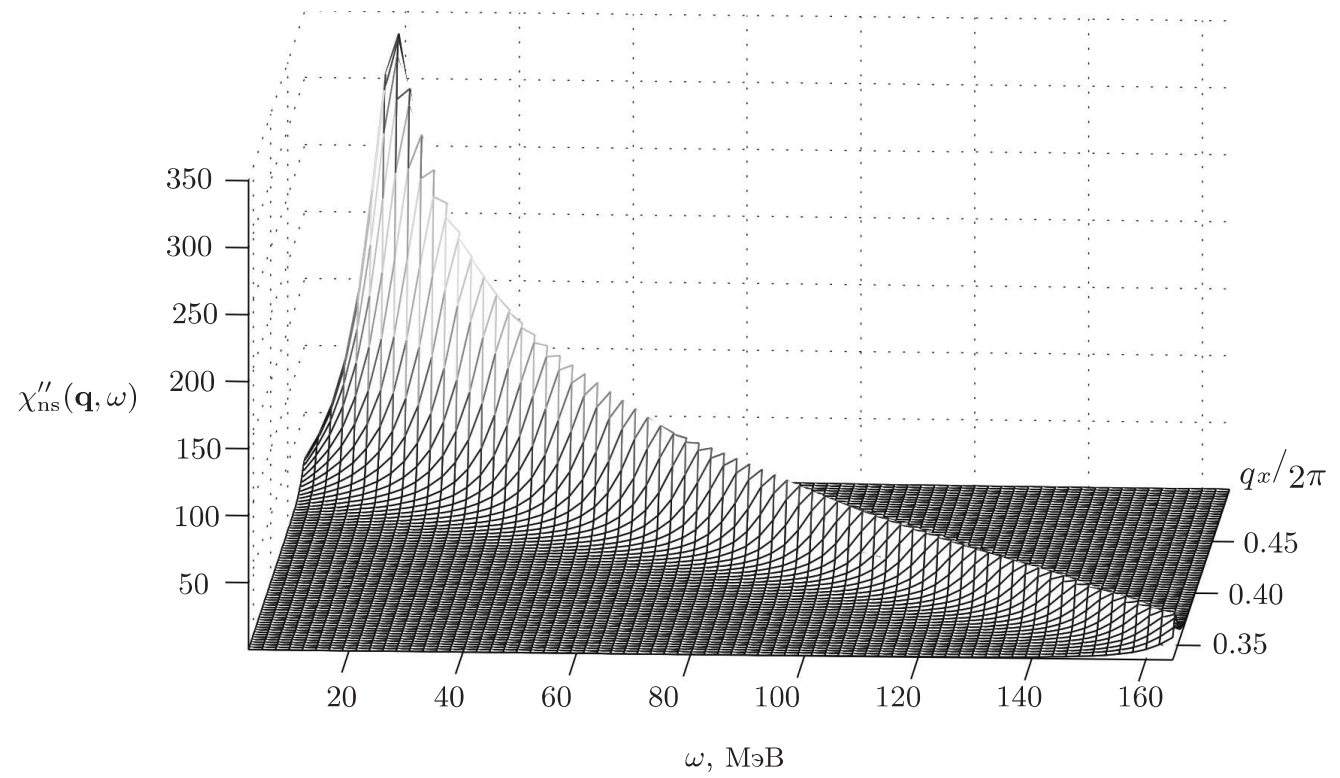

Рис. 11. Мнимая часть восприимчивости нормального состояния $\chi_{\mathrm{ns}}^{\prime \prime}(\mathbf{q}, \omega)$ в координатах $\left(q_{x}, \omega\right)$ для $\mathbf{q}-$ направления $q_{x}=q_{y}$ для значения фрустрации $p=0.15$ и параметров $J_{1}=(1-p) J=100 \mathrm{MэB}, T=0.01 J, \gamma=0.1 J$.

периментом представлены на рис. 10а, б. При этом считается, что значения $p=0.15$ и $p=0.28$ отвечают соответственно малому $(x \simeq 0.15)$ и большому $(x \simeq 0.5)$ допированию. Представленные результаты вновь поднимают вопрос об оценке величины внутриплоскостного обмена $J_{1}$ в иттриевых купратах. Наше рассмотрение показывает, что объяснение экспериментальной ситуации для $\mathrm{YBa}_{2} \mathrm{Cu}_{3} \mathrm{O}_{6+x}$ возможно без предположения об уменьшении $J_{1}$ на $60 \%$. K последнему предположению приводит подход линейных спиновых волн [42], который не контролирует выполнения правила сумм для $\chi^{\prime \prime}(\mathbf{q}, \omega)$.

Покажем теперь, что если ввести поляризационный оператор более сложного вида, чем $M_{\mathrm{ns}}^{\prime \prime}(\omega)=-\omega \gamma$, то возможно описание несоизмеримых пиков функции $\chi^{\prime \prime}(\mathbf{q}, \omega)$, которые возникают в случае допированной плоскости $\mathrm{CuO}_{2}$ при переходе дырочной системы в сверхпроводящее состояние. Будем считать, что $M_{\mathrm{ns}}^{\prime \prime}(\omega)$ отвечает нормальному состоянию. На рис. 11 представлена соответствующая функция $\chi_{\mathrm{ns}}^{\prime \prime}(\mathbf{q}, \omega)$ в координатах $\left(q_{x}, \omega\right)$ для q-направления $q_{x}=q_{y}$ для фрустрации $p=0.15$ и для следующих значений параметров: $J_{1}=(1-p) J=100 \mathrm{Mэ} \mathrm{B}, T=0.01 J, \gamma=0.1 J$.

В случае сверхпроводящего состояния в качестве приближения для $M(\mathbf{q}, \omega)$ выберем $M(\mathbf{q}, \omega)=i M_{\mathrm{ns}}^{\prime \prime}(\omega)+M_{\mathrm{scs}}(\mathbf{q}, \omega)$, где функция Грина $M_{\mathrm{scs}}(\mathbf{q}, \omega)=\Phi_{\mathbf{q}} /\left(\omega^{2}-\right.$ $\left.\varepsilon^{2}+i \omega \lambda\right)$ отвечает резонансному рассеянию на дырочной подсистеме, связанному с образованием сверхпроводящей щели на поверхности Ферми. Для коллективной ветви бездисперсионных спиновых возбуждений $M_{\mathrm{scs}}(\mathbf{q}, \omega)$ с энергией $\varepsilon$ и затуханием $\lambda$, которые возникают при переходе в сверхпроводящее состояние, разумно считать $\varepsilon \simeq 2 \bar{\Delta}_{\mathrm{sc}}$, где $\bar{\Delta}_{\mathrm{sc}}-$ усредненная сверхпроводящая щель (максимум $\left.2 \Delta_{\mathrm{sc}} \simeq 20-80 \mathrm{MэB}\right)$, т. е. мы полагаем, что резонанс связан с распадом куперовских 


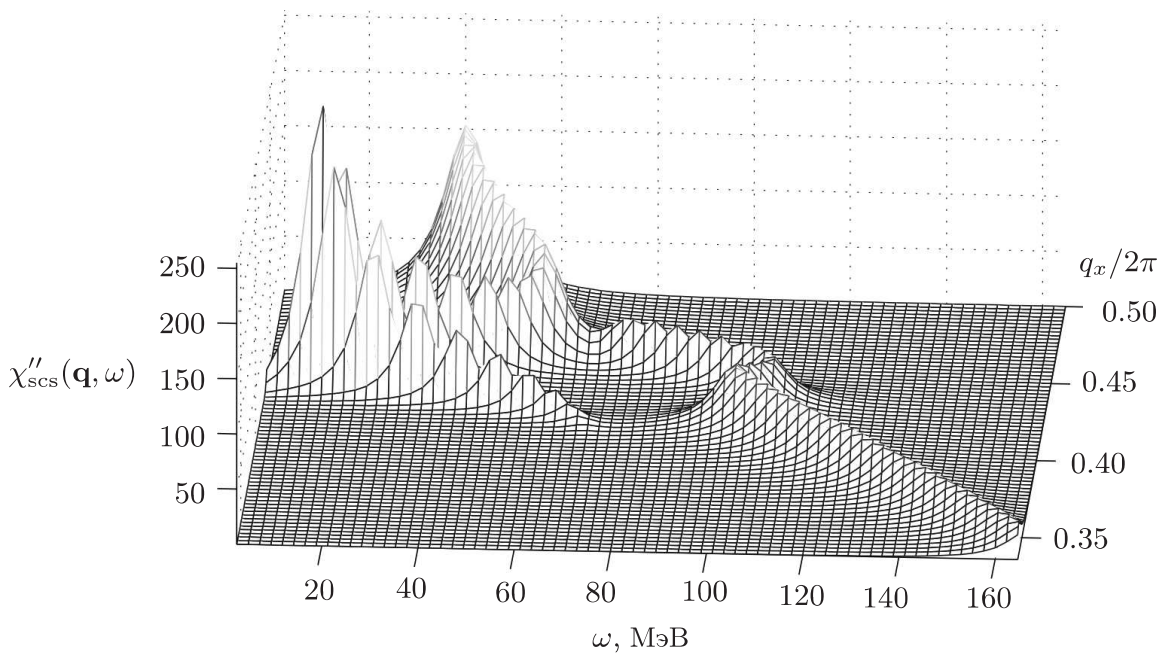

Рис. 12. Мнимая часть восприимчивости сверхпроводящего состояния $\chi_{\mathrm{scs}}^{\prime \prime}(\mathbf{q}, \omega)$ в координатах $\left(q_{x}, \omega\right)$ для $\mathbf{q}$-направления $q_{x}=q_{y}$ для значения фрустрации $p=0.15$ и параметров $J_{1}=(1-p) J=100 \mathrm{MэB}, T=0.01 J$, $\gamma=0.1 J, \varepsilon=0.6 J, \lambda=0.05 J$.

пар. Ниже мы положим $\varepsilon=0.6$, что отвечает величине, близкой к 70 МэВ. Модельно принятый числитель $\Phi_{\mathbf{q}}$ также должен иметь вид, обеспечивающий резонансное рассеяние между противоположными листами поверхности Ферми, т. е. максимум при $\mathbf{q}$, близком к $\mathbf{Q}=(\pi, \pi)$.

На рис. 12 представлена мнимая часть восприимчивости $\chi_{\mathrm{scs}}^{\prime \prime}(\mathbf{q}, \omega)$, найденная при прежних значениях $J_{1}, T, p, \gamma$, но с учетом $\varepsilon=0.6 J, \lambda=0.05 J$. Как видно, выход за простейшее приближение (15) приводит к возникновению дополнительной (относительно нормального состояния) ветви несоизмеримых пиков и к сильному отклонению от обычного спин-волнового поведения восприимчивости $\chi(\mathbf{q}, \omega)$ в области частот порядка 80 МэВ, так называемой “темной области”.

\section{4. ФАЗОВАЯ ДИАГРАММА МОДЕЛИ $J_{1}-J_{2}-J_{3}$}

Как отмечалось выше, рассмотрение модели $J_{1}-J_{2}-J_{3}$ представляет большой теоретический и экспериментальный интерес. В такой модели уже в классическом пределе реализуются четыре фазы, в том числе две несоизмеримые. В квантовом пределе возле границ этих фаз должна возникать область спиновой жидкости без LRO.

Разнообразие фаз с LRO и различных состояний спин-жидкостной фазы делает удобным пользоваться устоявшейся в численных работах терминологией [35], [51], [52]. Упоминавшиеся в разделе 1 NLRO- и StLRO-фазы в этой терминологии обозначаются соответственно как $\mathrm{LRO} \mathrm{C}(\pi, \pi)$ и LRO $\mathrm{C}(\pi, 0 ; 0, \pi)$ (символ $\mathrm{C}$ происходит от коллинеарных классических аналогов, координаты в скобках указывают положение точек, диктующих структуры дальнего порядка). 


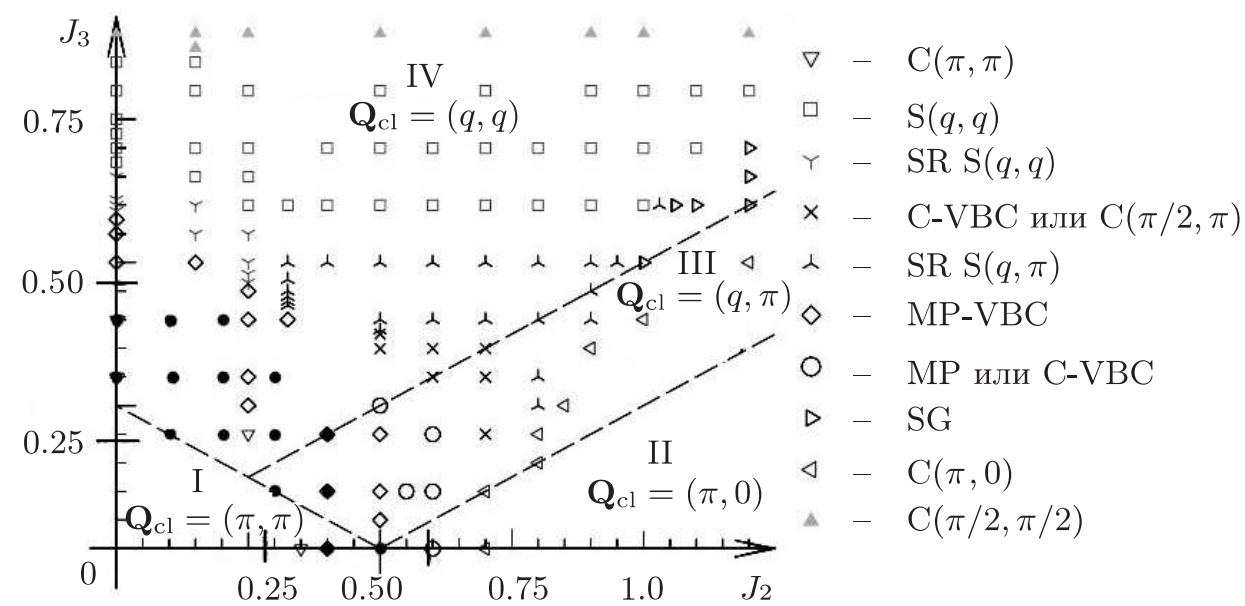

Рис. 13. Фазовая диаграмма основного состояния для $J_{\mathbf{1}}=1$. Символы справа отвечают рис. 2 работы [51] и соответствуют основным состояниям, найденным точной диагонализацией кластера с 32 узлами. Обозначения $\mathrm{C}(\pi, \pi), \mathrm{C}(\pi, 0), \mathrm{S}(q, q)$ отвечают в настоящей работе LRO-фазам $\mathrm{C}(\pi, \pi), \mathrm{LRO}$ $\mathrm{C}(\pi, 0 ; 0, \pi)$ и LRO $\mathrm{S}(k, k)$. Символы $\mathrm{SR} \mathrm{S}(q, q)$ и $\mathrm{SR} \mathrm{S}(q, \pi)$ отвечают состояниям спин-жидкостной фазы $\mathrm{SRO} \mathrm{S}(k, k)$ и $\mathrm{SRO} \mathrm{S}(k, \pi ; \pi, k)$. LRO-состояния, обозначенные в [51] $\mathrm{C}(\pi / 2, \pi)$ и $\mathrm{C}(\pi / 2, \pi / 2)$, в настоящей работе не рассматривались. В соответствии с [51] состояния, обозначенные остальными символами (MP, MP-VBC, C-VBC, SG), могут носить спин-жидкостный характер с нарушением трансляционной симметрии. Штриховые линии - классические границы LRO-фаз. Дополнительно к результатам [51] черными кружками отмечены точки, которые отвечают спин-жидкостной фазе работы [52] и для которых аналогичные состояния найдены в настоящей работе. На осях $J_{2}$ и $J_{3}$ длинными засечками отмечены найденные нами границы спин-жидкостной фазы.

В модели $J_{1}-J_{2}-J_{3}$, кроме LRO $\mathrm{C}(\pi, \pi)$ и LRO $\mathrm{C}(\pi, 0 ; 0, \pi)$, обсуждаются еще два LRO-состояния - LRO $\mathrm{S}(k, k)$ и LRO $\mathrm{S}(k, \pi ; \pi, k)$ с двумя типами геликоидального (спирального) несоизмеримого спинового порядка. Bce LRO-состояния являются отдельными фазами и обладают качественно различными LRO-типами, который диктуется точкой $\mathbf{q}_{0}$ обращения в нуль спиновой щели $\left(\mathbf{q}_{0}=(\pi, \pi),(\pi, 0 ; 0, \pi)\right.$, $(k, k),(k, \pi ; \pi, k))$. Кроме того, между LRO-состояниями возникает область спиновой жидкости, для которой спин-спиновые корреляторы на бесконечности равны нулю. В соответствии с доминированием для корреляторов того или иного типа SRO условно различают состояния $\mathrm{SRO} \mathrm{C}(\pi, \pi), \operatorname{SRO} \mathrm{C}(\pi, 0 ; 0, \pi), \operatorname{SRO} \mathrm{S}(k, k)$ и $\operatorname{SRO~} \mathrm{S}(k, \pi ; \pi, k)$. $\mathrm{B}$ нашем подходе все эти SRO-состояния отвечают одной и той же спин-жидкостной фазе. При приближении к границе существования LRO-фазы спиновая щель в соответствующей точке $\mathbf{q}_{0}$ зоны Бриллюэна стремится к нулю (а корреляционная длина - к бесконечности).

Стандартные спин-волновые вычисления позволяют вести рассмотрение только в областях, где существует LRO [53], [54]. В противоположность этому, здесь мы рассматриваем систему со стороны областей, где LRO и конденсат $c_{0}$ отсутствуют, 
a LRO-состояния диагностируются только на фазовых границах. Алгоритм нахождения функции Грина абсолютно аналогичен обсуждавшемуся выше, громоздкое выражение для $\omega_{\mathbf{q}}^{2}$ в этом случае содержит спин-спиновые корреляторы на первых восьми координационных сферах, и мы его не приводим. Результаты даны для значения $\gamma=0.6$. Везде ниже, в отличие от предыдущих разделов, параметр затухания $\gamma$ приводится в единицах $J_{1}+J_{2}+J_{3}$ (которые при $J_{3}=0$ переходят в $J$ для усеченной модели $\left.J_{1}-J_{2}\right)$. Приведенное значение $\gamma$ дает разумное согласие с кластерными расчетами как для положения границ фаз, так и для значений энергии. Все другие энергетические параметры приводятся в единицах $J_{1}, J_{1}=1$, как это принято в кластерных расчетах.

На рис. 13 вместе с границами классических фаз модели $J_{1}-J_{2}-J_{3}$ (штриховые линии) показаны результаты кластерных расчетов из работы [51] (см. рис. 2 в указанной работе). Разные символы отвечают различным спиновым структурам в рамках принятой в работе [51] классификации. Черными кружками отмечены точки, для которых в рамках кластерного расчета работы [52] (точная диагонализация кластера из 32 узлов) известна энергия. Для этих же точек в работе [52] предполагается отсутствие LRO. B настоящей работе в области этих точек находились функция Грина, спектр $\omega_{\mathbf{q}}$ и энергия. Наши результаты показывают, что в этой области система действительно находится в спин-жидкостной фазе.

Полученные энергии хорошо согласуются с данными кластерных расчетов [52]: расхождение в большинстве точек не превышает $10 \%$ и только вблизи точки фазового перехода $J_{3}=0, J_{2}=0.5$ достигает $14 \%$. Последнее связано с тем, что, по-видимому, эффективное затухание в этой области должно возрастать (в наших расчетах с ростом $\gamma$ от 0.6 до 1.0 и в этих точках расхождение не превышает 10\%). Тем не менее сравнение наших результатов с кластерными расчетами показывает, что приближение постоянного эффективного затухания $\gamma=0.6$ правильно отражает поведение системы в рассматриваемой области параметров.

Как отмечалось, в отличие от кластерных расчетов, СССП позволяет определить спектр спиновых возбуждений $\omega_{\mathbf{q}}$ и проследить величину и положение спиновой щели в зависимости от параметров обмена.

При движении в спин-жидкостной фазе по линии $J_{3}=0$, т. е. при увеличении $J_{2}$ от 0.28 до 0.60 (см. границы спин-жидкостной фазы на рис. 13), открывается и растет щель $\Delta_{\mathbf{Q}}$ в точке $\mathbf{Q}=(\pi, \pi)$, это означает переход из "шахматной" фазы LRO $\mathrm{C}(\pi, \pi)$ в спин-жидкостное состояние $\mathrm{SRO} \mathrm{C}(\pi, \pi)$. При этом с ростом $J_{2}$ уменьшается щель $\Delta_{\mathbf{X}}$ в точках $\mathbf{X}=(0, \pi),(\pi, 0)$. Закрытие щели в точке $\mathbf{X}$ означает переход из спин-жидкостного состояния $\operatorname{SRO} \mathrm{C}(\pi, 0 ; 0, \pi)$ в страйп-фазу $\operatorname{LRO} \mathrm{C}(\pi, 0 ; 0, \pi)$.

На рис. 14 представлена эволюция $\omega_{\mathbf{q}}$ в спин-жидкостной фазе вдоль линии $J_{2}=0$, т. е. при увеличении $J_{3}$ от 0.19 до 0.47 . Видно, что открывается и растет щель $\Delta_{\mathbf{Q}}$ в $\mathrm{A} \Phi \mathrm{M-точке} \mathbf{Q}=(\pi, \pi)$, т. е. происходит переход из “шахматной" фазы $\operatorname{LRO} \mathrm{C}(\pi, \pi)$ в спин-жидкостное состояние $\mathrm{SRO} \mathrm{C}(\pi, \pi)$. При этом с ростом $J_{3}$ уменьшается щель в несоизмеримой точке $\mathbf{q}_{0}=(k, k)$ на диагонали зоны Бриллюэна. Закрытие этой щели означает переход в геликоидальную фазу с несоизмеримым спиновым LRO $\mathrm{S}(k, k)$.

Итак, в настоящей работе при анализе спин-жидкостного состояния и его границ получены:

1) фазы LRO $\mathrm{C}(\pi, \pi)$, LRO $\mathrm{C}(\pi, 0 ; 0, \pi)$ и LRO $\mathrm{S}(k, k)$; в этих фазах, кроме тривиальной точки $q=0$, щель в спектре спиновых возбуждений закрыта в соответствующей точке $\mathbf{q}_{0}$ зоны Бриллюэна, спин-спиновый коррелятор на бесконечности 


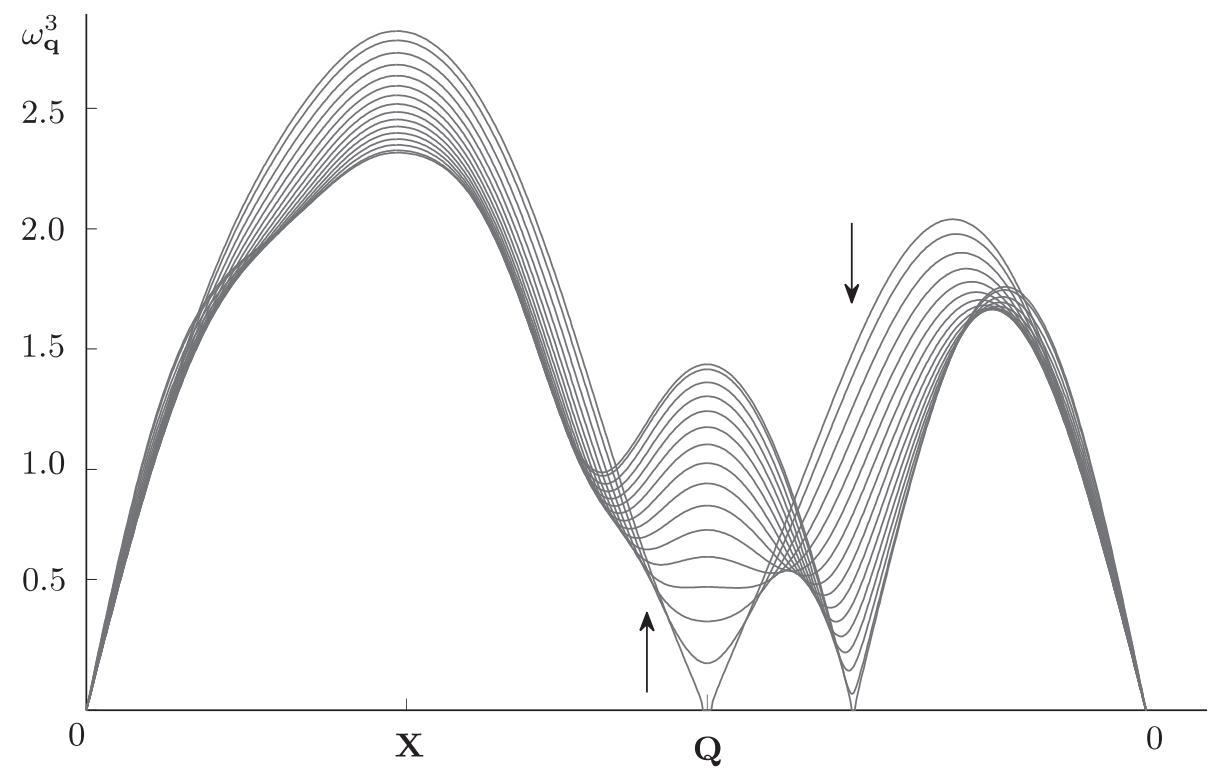

Рис. 14. Эволюция спектра спиновых возбуждений вдоль симметричных направлений в рамках СССП в области спин-жидкостной фазы при движении вдоль линии $J_{2}=0$ от границы фазы LRO $\mathrm{C}(\pi, \pi)$ до границы геликоидальной фазы LRO $\mathrm{S}(k, k)$ ( $\left.J_{3}=0.187-0.467\right)$. Стрелки указывают направление эволюции соответствующих участков спектра с ростом $J_{3}$. Параметр затухания $\gamma=0.6$.

отличен от нуля, а его характер определяется положением точки $\mathbf{q}_{0}$ (в этом разделе все LRO-фазы диагностированы на их границах со спиновой жидкостью);

2) спин-жидкостная фаза, в которой различаются области с доминированием различных $\mathrm{SRO-типов:} \mathrm{SRO} \mathrm{C}(\pi, \pi), \operatorname{SRO} \mathrm{C}(\pi, 0 ; 0, \pi), \operatorname{SRO} \mathrm{S}(k, k)$ и $\operatorname{SRO~} \mathrm{S}(k, \pi ; \pi, k)$; $\mathrm{SRO}$ диктуется положением наименьшей щели в спиновом спектре.

Отметим, что состояния $\mathrm{SRO} \mathrm{C}(\pi, \pi)$ и $\mathrm{SRO} \mathrm{C}(\pi, 0 ; 0, \pi)$ в работе [51] не диагностированы.

На рис. 15, 16 представлены полученные в настоящей работе типичные спектры спиновых возбуждений $\omega_{\mathbf{q}}$ на границах LRO-фаз (рис. 15 - страйп-фаза LRO $\mathrm{C}(\pi, 0 ; 0, \pi)$, рис. 16 - геликоид LRO $\mathrm{S}(k, k)$, типичный спектр в фазе LRO $\mathrm{C}(\pi, \pi)$ неоднократно приводился выше). Из рисунков видно, что щель в спиновом спектре закрыта в соответствующих точках зоны Бриллюэна: в точке $\mathbf{Q}=(\pi, \pi)$ в LRO $\mathrm{C}(\pi, \pi)$, в точках $\mathbf{X}=(0, \pi),(\pi, 0)$ в $\operatorname{LRO} \mathrm{C}(\pi, 0 ; 0, \pi)$ и в несоизмеримой точке $(k, k)$ на диагонали зоны Бриллюэна в LRO $\mathrm{S}(k, k)$.

Сравнение спектров показывает, что $\omega(\mathbf{q})$ имеет качественно различный вид в случае "шахматной”, страйп- и геликоидальной фаз. В частности, для геликоидальной фазы и страйп-фазы возникают дополнительные особенности Ван Хова, отвечающие почти бездисперсионному или квазиодномерному характеру спектра. В случае страйп-фазы (см. рис. 15) почти бездисперсионная область находится вблизи точки Q. В случае геликоидальной фазы (рис. 16) имеется линия, параллельная оси 


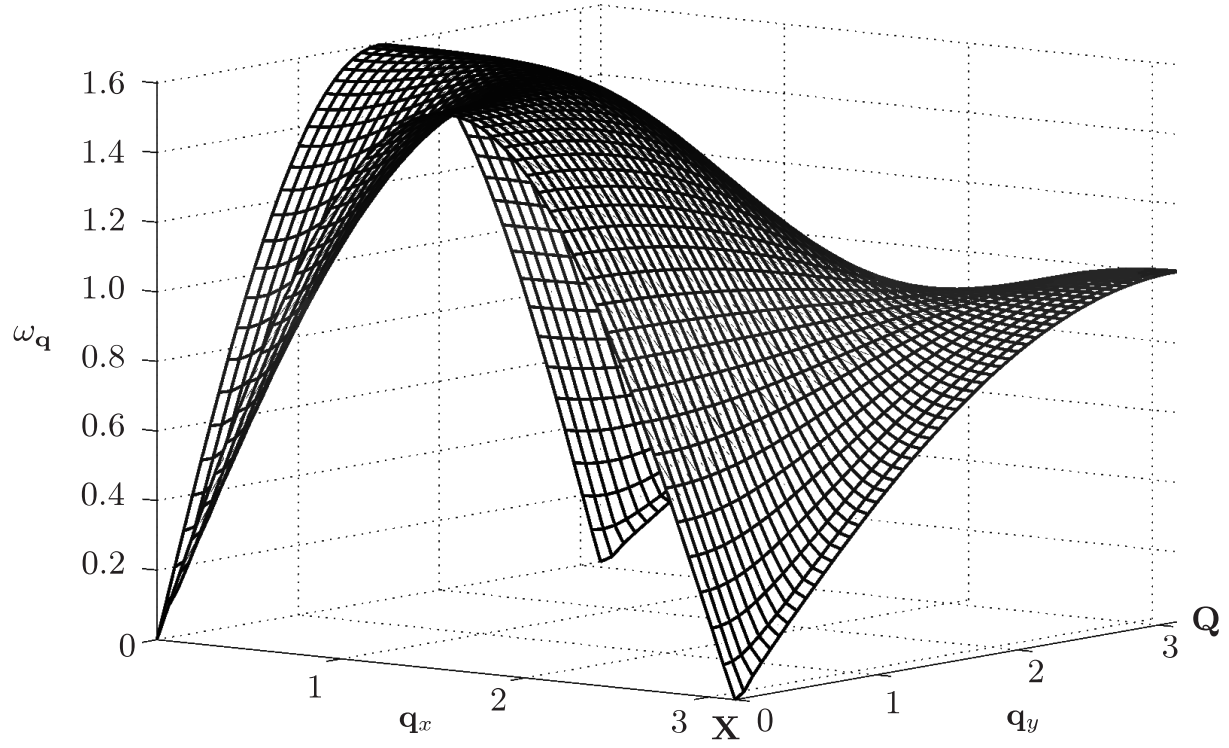

Рис. 15. Типичный спектр спиновых возбуждений $\omega_{\mathbf{q}}$ на границе страйп-фазы $\operatorname{LRO} \mathrm{C}(\pi, 0 ; 0, \pi)\left(J_{2}=0.6, J_{3}=0, \gamma=0.6\right)$. Щель закрыта в точках $\mathbf{X}=(0, \pi),(\pi, 0)$.

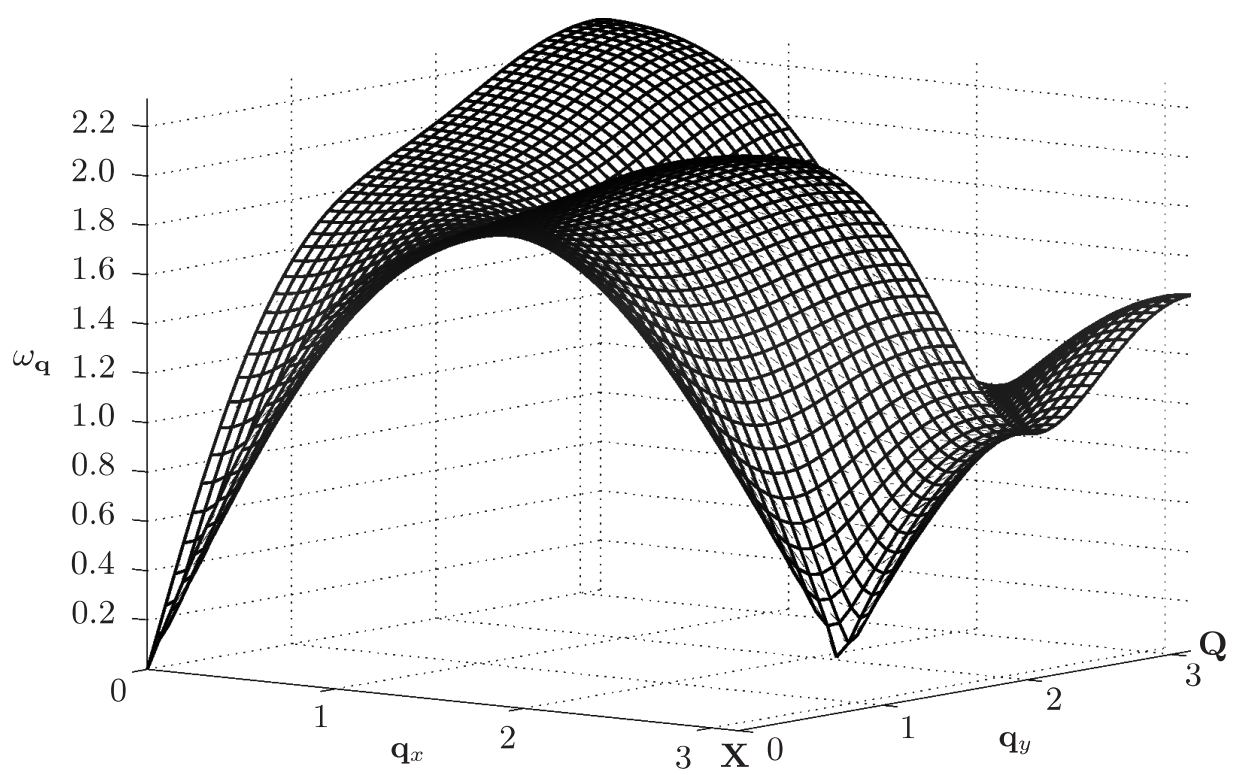

Рис. 16. Типичный спектр спиновых возбуждений $\omega_{\mathbf{q}}$ на границе геликоидальной фазы LRO $\mathrm{S}(k, k)\left(J_{2}=0, J_{3}=0.47, \gamma=0.6\right)$. Щель закрыта в несоизмеримой точке $(k, k)$. 
$\mathbf{X}-\mathbf{X}$, вдоль которой спектр бездисперсионен. Приведенные примеры спектра дают представление о нетривиальном характере его эволюции, отмеченной черными кружками на рис. 13 в области фазовой диаграммы. Что же касается существующей в классическом пределе четвертой LRO-фазы $\mathrm{S}(k, \pi ; \pi, k)$, то в области $J_{2}<2$, $J_{3}<2$ и при затухании $\gamma=0.6$ мы обнаружили только указание на ее возможное существование. В этой области CCCП дает геликоид без LRO, т. е. состояние с минимальной щелью в точке $(k, \pi)$. Отметим, что в кластерных расчетах [51] геликоид $(k, \pi)$ LRO также не обнаружен. С ростом затухания в наших расчетах геликоид $(k, \pi)$ появляется при $\gamma \sim 0.9$. Например, для $J_{2}=0.7, J_{3}=0.24, \gamma=0.86$ щель в несоизмеримой точке $(k, \pi)$ закрыта. Однако вопрос о восстановлении величины затухания в области существования геликоидальной фазы $(k, \pi)$ в настоящее время представляется открытым.

Таким образом, уже простейший учет затухания $\gamma$ позволяет реалистично описать многофазную ситуацию модели $J_{1}-J_{2}-J_{3}$.

\section{5. ЗАКЛЮЧЕНИЕ}

Наше рассмотрение указывает, прежде всего, на невозможность реалистического описания спиновой системы без учета затухания, даже при малых значениях параметра фрустрации. Только учет затухания позволяет получить скейлинговое поведение функции $\chi(\mathbf{q}, \omega, T)$, описать наблюдаемые в эксперименте спиновые щели и несоизмеримые пики. Особое значение при построении теории имеет аккуратный учет спинового констрейнта. Несмотря на то что уже простейшее приближение $M^{\prime \prime}=-\omega \gamma$ дает возможность отразить, например, даже сложную фазовую диаграмму модели $J_{1}-J_{2}-J_{3}$, представляется актуальным исследование перенормировок, связанных с действительной частью $M^{\prime}$ поляризационного оператора, и, конечно, как показывает наше рассмотрение несоизмеримых пиков, исследование зависимости $M$ от $\mathbf{q}$ и $\omega$. При изучении фазовой диаграммы модели $J_{1}-J_{2}-J_{3}$ представляется также интересным выйти за область фрустраций, рассмотренных в работе, в первую очередь, рассмотреть область с отрицательными значениями констант обмена и учесть зависимость $\gamma$ от фрустрации. Здесь можно ожидать реализации необычных фаз, когда в состоянии присутствуют одновременно два конденсата, отвечающих различным LRO.

Благодарности. Работа выполнена при поддержке РФФИ (грант № 10-02-00614).

\section{Список литературы}

[1] N. M. Plakida, High-Temperature Cuprate Superconductors: Experiment, Theory, and Applications, Springer Series in Solid-State Sciences, 166, Springer, Berlin, 2010.

[2] R. Melzi, P. Carretta, A. Lascialfari, M. Mambrini, M. Troyer, P. Millet, F. Mila, Phys. Rev. B, 85:6 (2000), 1318-1321.

[3] R. Nath, A. Tsirlin, H. Rosner, C. Geibell, Phys. Rev. B, 78:6 (2008), 64422, 7 pp., arXiv: 0803.3535.

[4] A. Tsirlin, H. Rosner, Phys. Rev. B, 79:21 (2009), 214417, 13 pp., arXiv: 0901.4498.

[5] M. Eschrig, Advances in Physics, 55:1 (2006), 47-183, arXiv: cond-mat/0510286.

[6] J.M. Tranquada, "Neutron Scattering Studies of Antiferromagnetic Correlations in Cuprates", Handbook of High-Temperature Superconductivity: Theory and Experiment, eds. J.R. Schrieffer, J.S. Brooks, Springer, New York, 2007, 257-298; arXiv: cond-mat/0512115. 
[7] J. Kondo, K. Yamaji, Prog. Theor. Phys., 47:3 (1972), 807-818.

[8] H. Shimahara, S. Takada, J. Phys. Soc. Jpn., 60:7 (1991), 2394-2405.

[9] A. F. Barabanov, O. A. Starykh, J. Phys. Soc. Jpn., 61:2 (1992), 704-708.

[10] J.F. Annet, R. M. Martin, A. K. McMahan, S. Satpathy, Phys. Rev. B, 40:4 (1989), $2620-2623$.

[11] M. Vojta, T. Ulbricht, Phys. Rev. Lett., 93:12 (2004), 127002, 4 pp., arXiv: cond-mat/0402377.

[12] А.Ф. Барабанов, В. М. Березовский, ЖКЭТФ, 106:4 (1994), 1156-1168; А. F. Barabanov,V. M. Berezovsky, J. Phys. Soc. Jpn., 63:11 (1994), 3974-3982.

[13] A. F. Barabanov, L. A. Maksimov, A. V. Mikheenkov, "Spin polaron in the cuprate superconductor: Interpretation of the ARPES results", Spectroscopy of High-Tc Superconductors. A Theoretical View, ed. N. M. Plakida, CRC Press, Boca Raton, 2003, 1-96.

[14] С. В. Тябликов, Методы квантовой теории магнетизма, Наука, М., 1975.

[15] A.F. Barabanov, A. V. Mikheyenkov, A. M. Belemuk, Phys. Lett. A, 365:5-6 (2007), 469-472.

[16] E. Manousakis, Rev. Mod. Phys., 63:1 (1991), 1-62.

[17] A. F. Barabanov, L. A. Maksimov, Phys. Lett. A, 207:6 (1995), 390-396.

[18] Ю. А. Церковников, ТМФ, 7:2 (1971), 250-261; 49:2 (1981), 219-233.

[19] N. M. Plakida, Phys. Lett. A, 43:6 (1973), 481-482.

[20] H. Mori, Progr. Theor. Phys., 33:3 (1965), 423-455; 34:4 (1965), 399-416.

[21] I. Sega, P. Prelovšek, J. Bonča, Phys. Rev. B, 68:5 (2003), 054524, 6 pp., arXiv: cond-mat/0211090.

[22] P. Prelovsek, I. Sega, J. Bonca, Phys. Rev. Lett., 92:2 (2004), 027002, 4 pp., arXiv: cond-mat/0306366.

[23] I. A. Larionov, Phys. Rev. B, 69:21 (2004), 214525, 17 pp., arXiv: cond-mat/0401514; 72:9 (2005), 094505, 5 pp.

[24] A. Sherman, Phys. Lett. A, 337:4-6 (2005), 435-440, arXiv: cond-mat/0501040.

[25] A. Sherman, M. Schreiber, Phys. Rev. B, 65:13 (2002), 134520, 8 pp., arXiv: cond-mat/0201526; 68:9 (2003), 094519, 8 pp., arXiv: cond-mat/0307195; Eur. Phys. J. B, 32:2 (2003), 203-214, arXiv: cond-mat/0302356.

[26] B. P. Stojković, D. Pines, Phys. Rev. B, 55:13 (1997), 8576-8595, arXiv: cond-mat/9609137.

[27] M. Millis, H. Monien, D. Pines, Phys. Rev. B, 42:1 (1990), 167-178.

[28] A.V. Chubukov, D.K. Morr, Electronic structure of underdoped cuprates, arXiv: cond-mat/9701196.

[29] S. Tyc, B. Halperin, Phys. Rev. B, 42:4 (1990), 2096-2115.

[30] A. A. Vladimirov, D. Ihle, N. M. Plakida, Phys. Rev. B, 80:10 (2009), 104425, 10 pp., arXiv: 0908.2557.

[31] А. М. Белемук, А.Ф. Барабанов, Л. А. Максимов, ЖЭТФ, 129:3 (2006), 493-506.

[32] А.Ф. Барабанов, Л. А. Максимов, Писъма в ЖЖЭТФ, 87:7 (2008), 433-437.

[33] А. В. Михеенков, А.Ф. Барабанов, ЖЭТФ, 132:2 (2007), 392-405.

[34] A. V. Mikheenkov, A. F. Barabanov, N. A. Kozlov, Phys. Lett. A, 354:4 (2006), 320-324.

[35] J. Richter, J. Schulenburg, Eur. Phys. J. B, 73:1 (2010), 117-124, arXiv: 0909.3723.

[36] M. A. Kastner, R. J. Birgeneau, G. Shirane, Y. Endoh, Rev. Mod. Phys., 70:3 (1998), 897-928.

[37] P. Bourges, "From Magnons to the Resonance Peak: Spin Dynamics in High-TC Superconducting Cuprates by Inelastic Neutron Scattering", The Gap Symmetry and Fluctuations in High-Tc Superconductors, Proceedings of a NATO ASI held in Cargese (France, September 1-13, 1997), NATO Science Series B: Physics, 371, eds. J. Bok, G. Deutscher, D. Pavuna, S. A. Wolf, Plenum, New York, 2002, 349-371, arXiv: cond-mat/9901333. 
[38] T. E. Mason, "Neutron Scattering Studies of Spin Fluctuations in High Temperature Superconductors", Handbook on the Physics and Chemistry of Rare Earths: High-Temperature Superconductors - II, 31, eds. J. K. A. Gschneidner, L. Eyring, M. B. Maple, Elsevier, Amsterdam, 2001, 281-314.

[39] R. J. Birgeneau, C. Stock, J. M. Tranquada et al., J. Phys. Soc. Jpn., 75:11 (2006), 111003, 14 pp., arXiv: cond-mat/0604667.

[40] J. Lorenzana, G. Seibold, R. Coldea, Phys. Rev. B, 72:22 (2005), 224511, 15 pp., arXiv: cond-mat/0507131.

[41] S. M. Hayden, G. Aeppli, H. A. Mook, T. G. Perring, T. E. Mason, S.-W. Cheong, Z. Fisk, Phys. Rev. Lett., 76:8 (1996), 1344-1347, arXiv: cond-mat/9611179.

[42] S. M. Hayden, G. Aeppli, T. G. Perring et al., Phys. Rev. B, 54:10 (1996), R6905-R6908.

[43] B. Keimer, R. J. Birgeneau, A. Cassanho, Y. Endoh, R. W. Erwin, M. A. Kastner, G. Shirane, Phys. Rev. Lett., 67 (1991), 1930-1933; B. Keimer, N. Belk, R. J. Birgeneau et al., Phys. Rev. B, 46:21 (1992), 14034-14053.

[44] K. Kakurai, S. Shamoto, T. Kiyokura, M. Sato, J. M. Tranquada, G. Shirane, Phys. Rev. B, 48:5 (1993), 3485-3490.

[45] H. Hiraka, Y. Endoh, M. Fujita, Y.S. Lee, J. Kulda, A. Ivanov, R. J. Birgeneau, J. Phys. Soc. Jpn., 70:3 (2001), 853-858, arXiv: cond-mat/0104087.

[46] C. Stock, W. J. L. Buyers, R. Liang, D. Peets, Z. Tun, D. Bonn, W. N. Hardy, R. J. Birgeneau, Phys. Rev. B, 69:1 (2004), 014502, 22 pp., arXiv: cond-mat/0308168.

[47] R. Coldea, S. M. Hayden, G. Aeppli, T. G. Perring, C. D. Frost, T. E. Mason, S.-W. Cheong, Z. Fisk, Phys. Rev. Lett., 86:23 (2001), 5377-5380, arXiv: cond-mat/0006384.

[48] J. Igarashi, Phys. Rev. B, 46:17 (1992), 107063-10771; J. Phys. Cond. Matt., 4:50 (1992), 10265-10276.

[49] R. R. P. Singh, Phys. Rev. B, 39:13 (1989), 9760-9763.

[50] Н. А. Козлов, А. Ф. Барабанов, Писъма в ЖКЭТФ, 85:11 (2007), 673-678.

[51] P. Sindzingre, N. Shannon, T. Momoi, J. Phys. Conf. Ser., 200:2 (2010), 022058, 4 pp., arXiv: 0907.4163.

[52] M. Mambrini, A. Lauchli, D. Poilblanc, F. Mila, Phys. Rev. B, 74:14 (2006), 144422, 11 pp., arXiv: cond-mat/0606776.

[53] A. Moreo, E. Dagotto, T. Jolicoeur, J. Riera, Phys. Rev. B, 42:10 (1990), 6283-6293.

[54] R. Rastelli, A. Tassi, Phys. Rev. B, 46:17 (1992), 10793-10799.

Поступила в редакцию 28.02.2011, после доработки 7.03.2011 